\title{
Structure of the human multidrug transporter ABCG2
}

\section{Journal Article}

\section{Author(s):}

Taylor, Nicholas M.I.; Manolaridis, Ioannis; Jackson, Scott M.; Kowal, Julia (D); Stahlberg, Henning; Locher, Kaspar P.

Publication date:

2017-06-22

Permanent link:

https://doi.org/10.3929/ethz-b-000233014

Rights / license:

In Copyright - Non-Commercial Use Permitted

\section{Originally published in:}

Nature 546(7659), https://doi.org/10.1038/nature22345 


\section{Structure of the human multidrug transporter ABCG2}

Nicholas M. I. Taylor ${ }^{* *}$, Ioannis Manolaridis ${ }^{2 *}$, Scott M. Jackson ${ }^{2 *}$, Julia Kowal ${ }^{2}$, Henning Stahlberg ${ }^{15}$ \& Kaspar P. Locher ${ }^{2 \$}$

* These authors contributed equally to this work

\$ Correspondence: henning.stahlberg@unibas.ch \& locher@mol.biol.ethz.ch

${ }^{1}$ Center for Cellular Imaging and NanoAnalytics (C-CINA), Biozentrum, University of Basel, Mattenstrasse 26, 4058 Basel, Switzerland

${ }^{2}$ Institute of Molecular Biology and Biophysics, ETH Zürich, Otto-Stern-Weg 5, 8093 Zurich, Switzerland 
ABCG2 is a constitutively expressed $\mathrm{ABC}$ transporter that protects many tissues against xenobiotic molecules. Its activity affects the pharmacokinetics of commonly used drugs and limits the delivery of therapeutics into tumor cells, thus contributing to multidrug resistance. We present the structure of human ABCG2 determined by cryo-electron microscopy, providing the first high-resolution insight into a human multidrug transporter. We visualized ABCG2 in complex with two antigen-binding fragments of the human-specific, inhibitory antibody 5D3 that recognizes extracellular loops of the transporter. We observed two cholesterol molecules bound in the multidrug-binding pocket that is located in a central, hydrophobic, inward-facing translocation pathway between the transmembrane domains. Combined with functional in vitro analyses, our results suggest a multidrug recognition and transport mechanism of ABCG2, rationalize disease-causing single nucleotide polymorphisms and the allosteric inhibition by the 5D3 antibody, and provide the structural basis of cholesterol recognition by other G-subfamily $\mathrm{ABC}$ transporters.

Humans have 48 distinct ATP-binding cassette (ABC) transporters that belong to seven subfamilies (A-G) and differ in size, architecture, and domain arrangement ${ }^{1,2}$. ABCG2, also known as Breast Cancer Resistance Protein (BCRP), is a multidrug transporter that has physiological roles in many tissues including the mammary gland and the blood-brain, blood-testis and maternal-foetal barriers $^{3-6}$. ABCG2 has a minor role in uric acid transport, but its dysfunction is associated with a number of disease states linked to hyperuricemia including gout, kidney disease and hypertension ${ }^{7}$. Studies with ABCG2 knockout mice have revealed the protein's impact on the oral availability of drugs in the gastrointestinal tract, whereas ABCG2 expressed in the canalicular membrane and in the apical membrane of kidney cells secretes diverse compounds into bile or urine. These two effects emphasize the impact of ABCG2 function on the pharmacokinetics of commonly used drugs. Consequently, ABCG2 is on the FDA (US Food and Drug Administration) and EMA (European Medicines Agency) lists of transporters to be checked for drug-drug interactions ${ }^{8}$. Like its functional homologues $\mathrm{ABCB} 1$ and $\mathrm{ABCC} 1, \mathrm{ABCG} 2$ also has a notorious function in extruding anti-tumor drugs from a variety of cancer cells, which can result in multidrug resistance (MDR), a serious obstacle in cancer treatment ${ }^{9-12}$. While its remarkable substrate polyspecificity and widespread localization make it a contributor to MDR, it has so far failed to emerge as a viable therapeutic target and thus an understanding of its drug-binding pocket is of paramount 
importance $e^{6,7,13-15}$. ABCG2 was also considered a marker for hematopoietic stem cells, and an inhibitory conformational antibody (5D3) against the human protein was developed ${ }^{16}$.

Over the past decades, in vivo studies have provided detailed insight into the localization, physiological functions, trafficking, substrate specificity, and single nucleotide polymorphisms of ABCG2 $2^{15-17}$. However, our mechanistic understanding of ABCG2 is limited because no highresolution structure is available, which is partly due to the challenges associated with its overexpression and functional purification. Whereas a crystal structure of the human sterol transporter ABCG5/G8 was recently reported ${ }^{18}$, direct structural insight into ABCG2 was limited to lowresolution electron microscopy imaging ${ }^{19}$ and an in silico model based on the structure of $\mathrm{ABCG} 5 / \mathrm{G}^{20}{ }^{20}$. In this study we employed single particle cryo-electron microscopy to determine the structure of wild-type, human ABCG2 in complex with two antigen binding fragments of 5D3 (5D3-Fab).

\section{Functional characterization and structure determination}

ABCG2 was expressed in human embryonic kidney cells (HEK293), purified in detergent solution, and reconstituted both into proteoliposomes and lipidic nanodiscs for functional studies. The reconstituted protein catalyzed transport of estrone-3-sulphate $\left(E_{1} S\right)$, a bona fide substrate of

$\mathrm{ABCG} 2$, into proteoliposomes, with $\mathrm{K}_{\mathrm{M}}$ values comparable to those previously reported using membrane vesicles derived from insect or human cells (Fig. 1a, b and Extended Data Fig. 1) ${ }^{21,22}$. $\mathrm{E}_{1} \mathrm{~S}$ transport was inhibited by $5 \mathrm{D} 3-\mathrm{Fab}$, but only if the antibody fragments were present in the lumen (inside) of the proteoliposomes. Thus our in vitro assays are consistent with earlier conclusions that $5 \mathrm{D} 3$ bound the extracellular surface of $\mathrm{ABCG} 2^{16}$. Transport of $\mathrm{E}_{1} \mathrm{~S}$ was completely abolished by the synthetic inhibitor Ko143 (Fig. 1b and Extended Data Fig. 1) ${ }^{23}$. Both 5D3-Fab and Ko143 reduced the ATPase activity of ABCG2 in liposomes while a mutation of the Walker-B residue E211 to a glutamine (E211Q) abolished activity (Fig. 1c).

Because nanodiscs contain a patch of lipid bilayer, they represent a physiologically relevant environment for structural studies of membrane proteins ${ }^{24,25}$. Negative stain EM revealed that two 5D3-Fabs bound to a single, nanodisc-reconstituted ABCG2 homodimer. The resulting ABCG25D3(Fab) complex had a molecular mass of $\sim 250 \mathrm{kDa}$ and maintained two-fold symmetry. Single particle cryo-EM analysis resulted in a three-dimensional reconstruction at an overall resolution of 
$3.8 \AA$ (Fourier shell correlation $=0.143$ criterion, Extended Data Fig. 2). The transmembrane domains (TMDs) and the variable domains (VD) of 5D3-Fab were better resolved, with local resolutions approaching $\sim 3 \AA$ (Extended Data Fig. 3). The quality of the EM density map allowed de novo building of an atomic model of the TMDs and of the VD part of 5D3-Fab (Extended Data Fig. 4). We independently determined the X-ray structure of 5D3-Fab at $1.5 \AA$ resolution (Extended Data Fig. 5), which provided an initial model to be docked into the EM density map. In contrast, the nucleotide-binding domains (NBDs) of ABCG2 were resolved at lower resolution, most likely due to their inherent flexibility and a lack of particle side views in the EM data. We therefore generated a homology model of the ABCG2 NBD based on the recent structure of ABCG5/G8 ${ }^{18}$ and docked this into our EM density map, where modifications to secondary structure elements could be fitted.

\section{Architecture of ABCG2}

Like other G-subfamily ABC transporters, $\mathrm{ABCG} 2$ is a "half-transporter" with one NBD and one TMD fused to a single polypeptide chain. The functional form of ABCG2 is a homodimer with a molecular mass of $\sim 144 \mathrm{kDa}$. In the ABCG2-5D3(Fab) complex, the Fabs bind at a $\sim 35^{\circ}$ angle relative to the membrane plane (Fig. 2a). The fold of ABCG2 (Fig. 2b, c) is similar to that of ABCG5/G8, but distinct from that found in the B-subfamily of ABC transporters, exemplified by the bacterial multidrug transporter homolog Sav $1866^{26}$ or the murine ABCB1 (P-glycoprotein) ${ }^{27,28}$. The TM helices and intracellular loops of ABCG2 are shorter compared to B-subfamily ABC transporters, resulting in a smaller distance between the NBDs and the membrane, and thus resembling the more compact bacterial $\mathrm{ABC}$ importers ${ }^{29,30}$. The TMD interface is formed by TM2 and TM5a of opposing ABCG2 monomers. The two $\mathrm{G}^{406} \mathrm{XXXG}^{410}$ motifs, previously postulated to be involved in dimerization ${ }^{31}$, are located on opposite sides of the transporter (Fig. 2b) and do not contact each other.

While the architecture of $A B C G 2$ is similar to that of $A B C G 5 / G 8$, there are significant differences that could not be predicted by the in silico model of $\mathrm{ABCG} 2^{20}$ due to model bias (r.m.s.d. between TMDs of our structure and the in silico model is $2.2 \AA$ ) (Extended Data Fig. 7b). First, there is a backbone shift in TM2 and TM5a of both monomers. This causes ABCG2 to form an inward- 
facing cavity (see below), a feature that is not present in ABCG5/G8. Second, the structure of the external loop EL3 is distinct in the two structures.

Intriguingly, the NBDs of ABCG2 remain in contact despite the absence of bound nucleotide. This was also observed in the structure of $\mathrm{ABCG} 5 / \mathrm{G} 8$ but differs from the large conformational flexibility reported for B-subfamily ABC transporters ${ }^{32-34}$. The NBD of each ABCG2 monomer is linked to TM1a (known in ABCG5/G8 as connecting helix - $\mathrm{CnH}$ ) via a highly charged linker. This linker is neither visible in the ABCG5/G8 X-ray structure nor in the ABCG2 EM density, most likely due to its flexibility, and therefore, it has not been modelled. The C2 motif of ABCG2, previously proposed to be important for ATP binding ${ }^{35}$, is located on an $\alpha$-helix at the bottom of the NBDs, $\sim 20 \AA$ away from the Walker A motif and $\sim 45 \AA$ from the signature motif, precluding its direct involvement in ATP binding to the canonical site (Fig. 2b).

\section{Interaction of inhibitory $5 \mathrm{D3}$ antibody with ABCG2}

Each 5D3-Fab interacts with extracellular loops (EL) of both ABCG2 monomers (Fig. 3a). Most contacts are formed by EL3, the longest of the extracellular loops in ABCG2, connecting TM5c and TM6a. EL3 contains three cysteines (C592, C603, C608) and a single N-glycosylation site (N596, Fig. 3b). Our map showed clear density for up to two GlcNAc residues attached to N596, and there is equally clear density for two intra-molecular disulfides (C592-C608 and C592'-C608') as well as one inter-molecular disulfide, C603-C603', which is positioned on the two-fold symmetry axis of the ABCG2 homodimer. The proper formation of the inter-molecular disulfide in our overexpression system was validated by SDS-PAGE, where fully dimeric ABCG2 was observed in non-reducing conditions both in the presence and absence of 5D3-Fab (Fig. 3c and Extended Data Fig. 6a). The intra-molecular disulfides stabilize the structure of EL3, which is in line with previous reports that $\mathrm{C} 592$ and $\mathrm{C} 608$ are important for ABCG2 maturation and activity $^{36,37}$. In contrast, the inter-molecular disulfide did not appear critical for transport function in cellular assays, as mutation to various residues abolished the formation of the covalent dimer, but did not interfere with drug extrusion ${ }^{38}$.

$\mathrm{N}$-glycosylation at N596 has been found to be essential for ABCG2 maturation, and mutations of this residue have resulted in protein destabilization and enhanced ubiquitin-mediated degradation ${ }^{39}$. N596 is not part of the ABCG2-5D3(Fab) interface, explaining why N-glycosylation of ABCG2 
does not affect the binding of 5D3 (Fig. 3b ${ }^{40}$. The physiological relevance of the interface observed in our structure is supported by the finding that $5 \mathrm{D} 3-\mathrm{Fab}$ is selective for human $\mathrm{ABCG} 2$ and does not bind to purified rat ABCG2 (Extended Data Fig. 6b). This can be rationalized by differences in the EL3 sequences of rat and human ABCG2, in particular those residues involved in the interface with 5D3 (N600, N601 and N604) (Fig. 3e). In addition, rat ABCG2 contains a second $\mathrm{N}$-glycosylation site (N600), which likely interferes with 5D3 binding.

The binding of two 5D3-Fabs is consistent with the two-fold symmetry of the ABCG2 homodimer. In contrast, only one Fab fragment of the inhibitory antibody UIC2 bound to the multidrug transporter $\mathrm{ABCB} 1$, which is asymmetric ${ }^{32}$. We investigated whether two copies of 5D3-Fab are required for inhibition. Using nanodisc-reconstituted ABCG2, we found that only one 5D3-Fab per ABCG2 homodimer is required to observe maximal inhibition of the ATPase activity (Fig. 3d). This suggests that a single 5D3 molecule can inhibit transport activity of ABCG2 by clamping the ABCG2 monomers together, precluding the formation of an outward-facing conformation that is essential for active transport. In addition, the inhibition of ATPase activity suggests relatively strict conformational coupling of the TMD and NBD motions, given that the antibody-binding site and

the ATPase sites are some $\sim 80 \AA$ apart and on opposite sides of the membrane. It was previously shown that whereas AMP, ADP, and ATP did not appear to affect 5D3 binding in vitro, binding of $5 \mathrm{D} 3$ was reduced by the presence of AMP-PNP or orthovanadate ${ }^{40}$.

\section{Translocation pathway and substrate binding site}

ABCG2 can bind or transport structurally diverse molecules ranging from hematoporphyrin, mitoxantrone, and methotrexate to inhibitors such as the tyrosine kinase inhibitor gefitinib or the fumitremorgin C-derived molecule Ko14323. The structure of ABCG2-5D3(Fab) revealed an inward-open conformation with a deep, slit-like cavity that is primarily lined by TM2 and TM5a from the two ABCG2 monomers. This cavity (cavity 1) opens to the cytoplasm and to the inner leaflet of the lipid bilayer and reaches more than halfway across the membrane to residues L554 and L554' of opposing ABCG2 monomers. These leucine residues form a plug that separates cavity 1 from a secondary, smaller cavity (cavity 2) located below the EL3s (Fig. 4a). Cavity 2 is inaccessible in the present conformation and is likely to have a lower affinity for substrate due to 
its less pronounced hydrophobic surface, which may help promote substrate release to the extracellular side during the transport cycle.

Given the size, hydrophobic nature, and accessibility of cavity 1 to the cytoplasm and the membrane, we assign it the role of a multidrug binding pocket. Its geometry makes it suitable to accommodate flat, polycyclic and hydrophobic substrates (Fig. 4b). We observed two large density features that were symmetrically located in cavity 1 . Their size and shape were inconsistent with bound phospholipids and suggested a polycyclic molecule (Fig. 4c, d). Only three such molecules were present during ABCG2 reconstitution into nanodiscs: cholesterol, cholesteryl hemisuccinate, and cholate. Of these, hemisuccinate could be excluded as a candidate because its substituents would cause a steric clash with the L554 plug at the top of cavity 1 . While cholate, used for the preparation of nanodiscs, would fit the density, functional data suggests that ABCG2 is not responsive to cholate in the presence or absence of $\mathrm{E}_{1} \mathrm{~S}$ (Extended Data Fig. 1f) We therefore interpreted the density features as two cholesterol molecules that are in contact with six hydrophobic residues from each ABCG2 monomer (F432 and F439 from TM2 and L539, I543, V546 and M549 from TM5a). The shape of the density guided us to fit the cholesterols with their hydroxyl groups pointing into the cavity, as the opposite orientation would generate a steric clash with the L554 plug (Extended Data Fig. 4e). We conclude that cholesterol, which is not a bona fide substrate, can nevertheless bind to ABCG2 and reveals where the chemically similar, physiological substrate $\mathrm{E}_{1} \mathrm{~S}$ would bind. Two $\mathrm{E}_{1} \mathrm{~S}$ molecules could indeed be fitted in the substrate binding pocket where the cholesterol molecules are bound and with their sulfate groups pointing towards the cytoplasm. The conclusion that cavity 1 represents an ABCG2 substrate-binding site is supported by the observation that the basal ATP hydrolysis rate of cholesterol-bound, nanodisc-reconstituted ABCG2 is indistinguishable from the $\mathrm{E}_{1} \mathrm{~S}$-stimulated ATPase activity in liposomes (Extended Data Fig. 1f).

While ABCG2 does not catalyze significant cholesterol transport in vivo, its function was previously shown to be regulated by cholesterol ${ }^{41}$. In contrast, the other members of the ABCGsubfamily $\left(\mathrm{ABCG} 1^{42}, \mathrm{ABCG} 4^{43}\right.$, and $\left.\mathrm{ABCG} 5 / \mathrm{G} 8\right)$ are reported sterol transporters. The residues in contact with bound cholesterol in ABCG2 are either fully conserved or substituted by other hydrophobic residues in the other G-subfamily ABC transporters, with the exception of V546, which corresponds to N568 in ABCG8 (Fig. 4e, f). Intriguingly, two of these six residues have been investigated in human ABCG5/G8. A mutation of Y432 in ABCG5 (corresponding to F439 
in $\mathrm{ABCG} 2$ ) to an alanine disrupted in vivo cholesterol transport ${ }^{18}$. Our structure reveals that $\mathrm{F} 439$ forms a stacking interaction with the B-ring of bound cholesterol (Fig. 4d). In addition, a mutation of A540 in ABCG5 (corresponding to V546 in ABCG2) to a phenylalanine also interfered with biliary cholesterol transport, which was interpreted as a possible steric clash with bound cholesterol by the larger, aromatic residue ${ }^{18}$. These results are fully in line with our structural data and validate our identification of a conserved sterol-binding cavity that is likely shared by other G-subfamily $\mathrm{ABC}$ transporters, even though the orientation of cholesterol may be different when recruited from the lipid bilayer (hydroxyl group pointing to the cytoplasm).

Cavity 2 observed in ABCG2 is not present in the ABCG5/G8 structure due to differences in the structure of EL3. The sequence similarity in EL3 is low among ABCG-subfamily members (Extended Data Fig. 7c). Whereas ABCG1, ABCG4 and ABCG5 each have two cysteines in similar positions to ABCG2 (C587, C600), ABCG8 has no cysteines in EL3. It is conceivable that the formation of intra-molecular disulfides in EL3 of ABCG2 contributes to its function as a multidrug transporter, because EL3 is a key element in forming cavity 2 .

\section{Single nucleotide polymorphisms of ABCG2}

The mutation Q141K is an ABCG2 single nucleotide polymorphism (SNP) that is linked to hyperuricemia and gout ${ }^{44}$. It results in decreased ABCG2 expression, misfolding and degradation by the ERAD system. Nevertheless, residual ABCG2 (Q141K) in the plasma membrane shows transport activity ${ }^{45}$. Despite the lower resolution in the NBDs of our ABCG2 structure, we can place Q141 on the $\alpha$-helix adjacent to TM1a (Fig. 2b). TM1a is an amphipathic $\alpha$-helix, and its positively charged side faces Q141. We expect that the mutation of Q141 to a positively charged lysine would result in electrostatic repulsion that could distort TM1a, affecting ABCG2 folding. Another SNP-associated residue, R482, has been extensively studied and reported to be important for $\mathrm{ABCG} 2$ function. Its mutation to various residues affects substrate specificity ${ }^{46}$, although it was proposed that this is not through direct substrate interaction ${ }^{47}$. In our structure, R482 is located on TM3 (Fig. 2b) where it forms a stabilizing hydrogen bond with S521 on TM4, but is approximately $9 \AA$ away from the substrate-binding pocket. The reported impact on substrate specificity is therefore likely caused by allosteric effects. 


\section{Proposed transport mechanism}

Our results allow us to formulate a transport and inhibition mechanism that is consistent with the available biochemical and functional data. The mechanism invokes ATP-driven alternating access $^{48}$, whereby the closing of the NBD interface in response to ATP binding converts the substrate-bound, inward-open conformation of ABCG2 to an outward-facing state that can release the substrate to the outside (Fig. 5). In the absence of bound ATP, the hydrophobic substratebinding site (cavity 1) is exposed to the cytoplasm and the inner leaflet of the plasma membrane, from where substrate is recruited. In this state, cavity 2 is empty but occluded. Upon binding of substrate and ATP, conformational changes convert the transporter to an outward-facing state, where the substrate is moved into cavity 2 , from where it is expelled into the extracellular medium by means of a hydrophobic mismatch. The transporter cannot convert to an inward-facing state as long as the substrate is present in cavity 2 due to steric clashes. Thus, ATP hydrolysis and the conversion to an inward-facing state are directly linked to substrate release, providing the molecular basis of active transport. The inward-facing state can be inhibited by 5D3-Fab. Although two 5D3-Fabs can bind to the extracellular side, only one is required to clamp the ABCG2 monomers together, restricting their relative motion and allosterically impairing both substrate transport and ATP hydrolysis by shifting the equilibrium to the inward-facing conformation. This demonstrates strong coupling between TMDs and NBDs in ABCG2, which in turn can provide a molecular explanation for substrate-induced ATPase stimulation: substrate binding to cavity 1 probably stimulates the ATPase activity of the transporter by stabilizing the TMD interface, which results in closer proximity of the NBDs. The structure presented here reveals that inhibitory 5D3Fab and substrate binding are not mutually exclusive, but transport from this state is impossible as long as the TMDs are externally clamped together by the antibody fragments.

\section{Conclusions}

The ABCG2 structure represents the first high-resolution structure of a human multidrug ABC transporter. The observed geometry and architecture of the hydrophobic substrate-binding cavity provides the structural basis of the polyspecific interaction with diverse hydrophobic compounds and offers an opportunity to model drugs and inhibitors into the multidrug binding pocket, which may facilitate structure-based drug and inhibitor design. Our results also define the mechanism by 
which an inhibitory antibody, through long-range allosteric effects, precludes conformational changes that are essential for substrate transport. While probing of additional drug- and inhibitorbound states is essential for a complete mechanistic understanding, our results can already be used for diagnostic and potential therapeutic approaches.

\section{References}

1 Moitra, K. \& Dean, M. Evolution of ABC transporters by gene duplication and their role in human disease. Biological chemistry 392, 29-37, (2011).

2 Dean, M., Rzhetsky, A. \& Allikmets, R. The human ATP-binding cassette (ABC) transporter superfamily. Genome research 11, 1156-1166, (2001).

3 Cuperus, F. J., Claudel, T., Gautherot, J., Halilbasic, E. \& Trauner, M. The role of canalicular $\mathrm{ABC}$ transporters in cholestasis. Drug metabolism and disposition: the biological fate of chemicals 42, 546-560, (2014).

4 Fetsch, P. A. et al. Localization of the ABCG2 mitoxantrone resistance-associated protein in normal tissues. Cancer letters 235, 84-92, (2006).

5 Diestra, J. E. et al. Frequent expression of the multi-drug resistance-associated protein BCRP/MXR/ABCP/ABCG2 in human tumours detected by the BXP-21 monoclonal antibody in paraffin-embedded material. The Journal of pathology 198, 213-219, (2002).

6 Robey, R. W. et al. ABCG2: a perspective. Advanced drug delivery reviews 61, 3-13, (2009).

7 Ishikawa, T., Aw, W. \& Kaneko, K. Metabolic Interactions of Purine Derivatives with Human ABC Transporter ABCG2: Genetic Testing to Assess Gout Risk.

Pharmaceuticals 6, 1347-1360, (2013).

8 Hillgren, K. M. et al. Emerging transporters of clinical importance: an update from the International Transporter Consortium. Clinical pharmacology and therapeutics 94, 52-63, (2013).

9 Sarkadi, B., Homolya, L., Szakacs, G. \& Varadi, A. Human multidrug resistance ABCB and ABCG transporters: participation in a chemoimmunity defense system. Physiological reviews 86, 1179-1236, (2006).

10 Gottesman, M. M., Fojo, T. \& Bates, S. E. Multidrug resistance in cancer: role of ATPdependent transporters. Nature reviews. Cancer 2, 48-58, (2002).

11 Sharom, F. J. The P-glycoprotein multidrug transporter. Essays in biochemistry 50, 161178, (2011).

12 Gillet, J. P. \& Gottesman, M. M. Advances in the molecular detection of ABC transporters involved in multidrug resistance in cancer. Current pharmaceutical biotechnology 12, 686-692, (2011).

13 Mo, W. \& Zhang, J. T. Human ABCG2: structure, function, and its role in multidrug resistance. International journal of biochemistry and molecular biology 3, 1-27, (2012).

14 Robey, R. W., Ierano, C., Zhan, Z. \& Bates, S. E. The challenge of exploiting ABCG2 in the clinic. Current pharmaceutical biotechnology 12, 595-608, (2011).

15 Tamaki, A., Ierano, C., Szakacs, G., Robey, R. W. \& Bates, S. E. The controversial role of ABC transporters in clinical oncology. Essays in biochemistry 50, 209-232, (2011). 
16 Zhou, S. et al. The ABC transporter Bcrp1/ABCG2 is expressed in a wide variety of stem cells and is a molecular determinant of the side-population phenotype. Nature medicine 7 , 1028-1034, (2001).

17 Robey, R. W., Massey, P. R., Amiri-Kordestani, L. \& Bates, S. E. ABC transporters: unvalidated therapeutic targets in cancer and the CNS. Anti-cancer agents in medicinal chemistry 10, 625-633, (2010).

18 Lee, J. Y. et al. Crystal structure of the human sterol transporter ABCG5/ABCG8. Nature 533, 561-564, (2016).

19 Rosenberg, M. F. et al. Three-dimensional structure of the human breast cancer resistance protein (BCRP/ABCG2) in an inward-facing conformation. Acta crystallographica.

Section D, Biological crystallography 71, 1725-1735, (2015).

20 Laszlo, L., Sarkadi, B. \& Hegedus, T. Jump into a New Fold-A Homology Based Model for the ABCG2/BCRP Multidrug Transporter. PloS one 11, e0164426, (2016).

21 Suzuki, M., Suzuki, H., Sugimoto, Y. \& Sugiyama, Y. ABCG2 transports sulfated conjugates of steroids and xenobiotics. The Journal of biological chemistry 278, 2264422649, (2003).

22 Karlsson, J. E. et al. High-activity p-glycoprotein, multidrug resistance protein 2, and breast cancer resistance protein membrane vesicles prepared from transiently transfected human embryonic kidney 293-epstein-barr virus nuclear antigen cells. Drug metabolism and disposition: the biological fate of chemicals 38, 705-714, (2010).

23 Allen, J. D. et al. Potent and specific inhibition of the breast cancer resistance protein multidrug transporter in vitro and in mouse intestine by a novel analogue of fumitremorgin C. Molecular cancer therapeutics 1, 417-425, (2002).

24 Ritchie, T. K. et al. Chapter 11 - Reconstitution of membrane proteins in phospholipid bilayer nanodiscs. Methods in enzymology 464, 211-231, (2009).

25 Nath, A., Atkins, W. M. \& Sligar, S. G. Applications of phospholipid bilayer nanodiscs in the study of membranes and membrane proteins. Biochemistry 46, 2059-2069, (2007).

26 Dawson, R. J. \& Locher, K. P. Structure of a bacterial multidrug ABC transporter. Nature 443, 180-185, (2006).

27 Aller, S. G. et al. Structure of P-glycoprotein reveals a molecular basis for poly-specific drug binding. Science 323, 1718-1722, (2009).

28 Li, J., Jaimes, K. F. \& Aller, S. G. Refined structures of mouse P-glycoprotein. Protein science : a publication of the Protein Society 23, 34-46, (2014).

29 Locher, K. P., Lee, A. T. \& Rees, D. C. The E. coli BtuCD structure: a framework for ABC transporter architecture and mechanism. Science 296, 1091-1098, (2002).

30 Woo, J. S., Zeltina, A., Goetz, B. A. \& Locher, K. P. X-ray structure of the Yersinia pestis heme transporter HmuUV. Nature structural \& molecular biology 19, 1310-1315, (2012).

31 Polgar, O. et al. Mutational analysis of ABCG2: role of the GXXXG motif. Biochemistry 43, 9448-9456, (2004).

32 Frank, G. A. et al. Cryo-EM Analysis of the Conformational Landscape of Human Pglycoprotein (ABCB1) During its Catalytic Cycle. Molecular pharmacology 90, 35-41, (2016).

33 Shintre, C. A. et al. Structures of ABCB10, a human ATP-binding cassette transporter in apo- and nucleotide-bound states. Proceedings of the National Academy of Sciences of the United States of America 110, 9710-9715, (2013).

34 Perez, C. et al. Structure and mechanism of an active lipid-linked oligosaccharide flippase. Nature 524, 433-438, (2015). 
35 Macalou, S. et al. The linker region of breast cancer resistance protein ABCG2 is critical for coupling of ATP-dependent drug transport. Cellular and molecular life sciences : CMLS 73, 1927-1937, (2016).

36 Henriksen, U., Fog, J. U., Litman, T. \& Gether, U. Identification of intra- and intermolecular disulfide bridges in the multidrug resistance transporter $\mathrm{ABCG} 2$. The Journal of biological chemistry 280, 36926-36934, (2005).

37 Wakabayashi, K. et al. Identification of cysteine residues critically involved in homodimer formation and protein expression of human ATP-binding cassette transporter ABCG2: a new approach using the flp recombinase system. J Exp Ther Oncol 5, 205-222, (2006).

38 Kage, K., Fujita, T. \& Sugimoto, Y. Role of Cys-603 in dimer/oligomer formation of the breast cancer resistance protein BCRP/ABCG2. Cancer science 96, 866-872, (2005).

39 Nakagawa, H. et al. Disruption of N-linked glycosylation enhances ubiquitin-mediated proteasomal degradation of the human ATP-binding cassette transporter ABCG2. The FEBS journal 276, 7237-7252, (2009).

40 Ozvegy-Laczka, C. et al. Function-dependent conformational changes of the ABCG2 multidrug transporter modify its interaction with a monoclonal antibody on the cell surface. The Journal of biological chemistry 280, 4219-4227, (2005).

41 Telbisz, A., Hegedus, C., Varadi, A., Sarkadi, B. \& Ozvegy-Laczka, C. Regulation of the function of the human ABCG2 multidrug transporter by cholesterol and bile acids: effects of mutations in potential substrate and steroid binding sites. Drug metabolism and disposition: the biological fate of chemicals $\mathbf{4 2}, 575-585$, (2014).

42 Tarling, E. J. \& Edwards, P. A. ATP binding cassette transporter G1 (ABCG1) is an intracellular sterol transporter. Proceedings of the National Academy of Sciences of the United States of America 108, 19719-19724, (2011).

43 Tarr, P. T. \& Edwards, P. A. ABCG1 and ABCG4 are coexpressed in neurons and astrocytes of the CNS and regulate cholesterol homeostasis through SREBP-2. Journal of lipid research 49, 169-182, (2008).

44 Basseville, A. \& Bates, S. E. Gout, genetics and ABC transporters. F1000 biology reports 3, 23, (2011).

45 Furukawa, T. et al. Major SNP (Q141K) variant of human ABC transporter ABCG2 undergoes lysosomal and proteasomal degradations. Pharmaceutical research 26, 469479, (2009).

46 Honjo, Y. et al. Acquired mutations in the MXR/BCRP/ABCP gene alter substrate specificity in MXR/BCRP/ABCP-overexpressing cells. Cancer research 61, 6635-6639, (2001).

47 Ejendal, K. F., Diop, N. K., Schweiger, L. C. \& Hrycyna, C. A. The nature of amino acid 482 of human ABCG2 affects substrate transport and ATP hydrolysis but not substrate binding. Protein science : a publication of the Protein Society 15, 1597-1607, (2006).

48 Locher, K. P. Mechanistic diversity in ATP-binding cassette (ABC) transporters. Nature structural \& molecular biology 23, 487-493, (2016).

\section{Acknowledgements}


This research was supported by the Swiss National Science Foundation through NCCR TransCure, and by ETH research grant ETH-22-14-1. We thank the staff of the X06SA beamline of the Swiss Light Source for support during data collection. We thank Kenneth Goldie, Ariane FecteauLeFebre, and Robert McLeod for support during electron microscope usage, and Ricardo Adaixo, Lena Muckenfuss, Nikhil Biyani, and Ricardo Righetto for support and discussions during EM data analysis. We thank Loick Lancien and Bernadette Prinz for help with protein expression and cell culture work. We thank Brian Sorrentino for providing the 5D3-producing hybridoma cell line, Bruno Stieger for helpful discussions regarding transport assays, and Jung-In Kim for initial ABCG2 expression and purification experiments.

\section{Author contributions}

I.M. expressed human and rat ABCG2. I.M. and S.M.J. purified and reconstituted ABCG2 in liposomes and nanodiscs. S.M.J. performed functional assays. J.K. performed initial negative stain and cryo-EM analyses and prepared all grids. N.M.I.T. and H.S. performed cryo-EM data collection. N.M.I.T. performed ABCG2 structure determination and built the ABCG2 model. I.M., K.P.L. and N.M.I.T. revised the model, and N.M.I.T. performed model refinement. I.M. determined the 5D3-Fab crystal structure. K.P.L., I.M., S.M.J., and H.S. conceived the project and planned the experiments. I.M., S.M.J. and K.P.L. wrote the manuscript, all authors contributed to manuscript revision.

\section{Author Information}

Reprints and permissions information is available at www.nature.com/reprints. The authors declare no competing financial interests. Readers are welcome to comment on the online version of the paper. Correspondence and requests for materials should be addressed to H.S. (henning.stahlberg@unibas.ch) and K.P.L. (locher@mol.biol.ethz.ch). 


\section{METHODS}

No statistical methods were used to predetermine sample size. The experiments were not randomized. The investigators were not blinded to allocation during experiments and outcome assessment.

\section{Expression and purification of human ABCG2}

Human full-length ABCG2 (Uniprot: Q9UNQ0), containing an N-terminal Flag tag, was expressed in HEK293-EBNA cells as previously described ${ }^{49}$. The cells were treated with $5 \mu \mathrm{M}$ kifunensine (Toronto Research Chemicals) for 30 min prior to transfection. Following transfection the cells were incubated for 2 days at $37^{\circ} \mathrm{C}$ before harvesting.

Transfected cells were solubilized in $40 \mathrm{mM}$ HEPES pH 7.5, $150 \mathrm{mM} \mathrm{NaCl}, 10 \% \mathrm{v} / \mathrm{v}$ glycerol, 1 $\mathrm{mM}$ PMSF, $2 \mu \mathrm{g} \mathrm{mL}^{-1}$ DNaseI (Roche) and protease inhibitor cocktail (Sigma) with 1\% DDM + $0.1 \%$ CHS (w/v) (Anatrace) for $90 \mathrm{~min}$ at $4{ }^{\circ} \mathrm{C}$. The solubilized lysate was ultracentrifuged at $100,000 \mathrm{~g}$ and the supernatant was incubated with anti-Flag M2 affinity agarose gel (Sigma). The gel was washed extensively with purification buffer containing $40 \mathrm{mM}$ HEPES pH 7.5, $150 \mathrm{mM}$ $\mathrm{NaCl}$ and $0.026 \% \mathrm{DDM}+0.0026 \% \mathrm{CHS}(\mathrm{w} / \mathrm{v})$. ABCG2 was eluted with Flag peptide (Sigma) and treated with EndoF before being loaded into a TSKgel G3000SWXL column (Tosoh Bioscience). The peak fractions were collected and reconstituted into nanodiscs or liposomes and used for either cryo-EM studies or functional assays.

\section{ABCG2-liposome preparation for transport and ATPase assays}

A brain polar lipid:cholesterol (BPL:chol) (4:1) (w/w) mixture was prepared as described previously ${ }^{50}$. Briefly, the BPL:chol mixture was extruded 11 times through a $400 \mathrm{~nm}$ filter and destabilized with $0.17 \% \mathrm{v} / \mathrm{v}$ Triton-X-100. Detergent-purified ABCG2 was then mixed with BPL:chol at a 100:1 (w/w) lipid:protein ratio. Detergent was removed with Bio-Beads and proteoliposomes were spun at 100,000g, resuspended in $25 \mathrm{mM}$ HEPES $\mathrm{pH} 7.5,150 \mathrm{mM} \mathrm{NaCl}$ at a final lipid concentration of $10 \mathrm{mg} \mathrm{mL}^{-1}$ and the reconstitution efficiency was determined ${ }^{51}$. 


\section{ABCG2-nanodisc preparation for ATPase assays}

Membrane scaffold protein (MSP) 1D1 was expressed and purified as described previously ${ }^{24}$. BPL:CHS (4:1) (w/w) was solubilized with a $3 \times$ Molar excess of sodium cholate using a sonic bath. Solubilized BPL:CHS was mixed with MSP 1D1 and detergent-purified ABCG2 at a Molar ratio of 100:5:0.2 (lipid:MSP:ABCG2). Bio-Beads were added and the sample was incubated at 4 ${ }^{\circ} \mathrm{C}$ overnight. Bio-Beads were removed and the sample was spun at 100,000 $\mathrm{g}$ before being loaded into a Superdex 200 10/300 column (GE Healthcare) and used either for cryo-EM studies or ATPase assays.

\section{Transport assays and $\mathrm{E}_{1} \mathrm{~S} \mathrm{~K}_{\mathrm{M}}$ determination}

Liposome-reconstituted ABCG2 was freeze-thawed (FT) in the presence or absence of a $3 \times$ Molar excess of 5D3-Fab to get the 5D3-Fab inside the proteoliposomes. FT was not detrimental to the function of ABCG2. Proteoliposomes were then extruded through a $400 \mathrm{~nm}$ polycarbonate filter and diluted to $4 \mathrm{mg} \mathrm{mL}^{-1}$ lipid in transport buffer (25 mM HEPES pH 7.5, $150 \mathrm{mM} \mathrm{NaCl}$ ). $5 \mathrm{mM}$ $\mathrm{MgCl}_{2}$ and $1-50 \mu \mathrm{M}{ }^{3} \mathrm{H}$-estrone-3-sulfate $\left({ }^{3} \mathrm{H}-\mathrm{E}_{1} \mathrm{~S}\right)$ were added in the presence or absence of 5D3Fab or Ko143 and the samples incubated for $5 \mathrm{~min}$ at $30^{\circ} \mathrm{C}$. The transport reaction was initiated by the addition of $2 \mathrm{mM}$ ATP. To stop the reaction samples were removed, added to ice-cold transport buffer containing unlabelled $\mathrm{E}_{1} \mathrm{~S}$, filtered using a Multiscreen vacuum manifold (MSFBN6B filter plate, Millipore) and washed three times. Radioactivity trapped on the filters was measured using a Perkin Elmer 2450 Microbeta2 microplate scintillation counter. Data were analyzed and curves were plotted using the non-linear regression Michaelis-Menten analysis tool and initial rates were determined using linear-regression in GraphPad Prism 7 (GraphPad Software, La Jolla California USA).

\section{ATPase assays and stoichiometry}

ATP hydrolysis activity was assessed as described previously ${ }^{52}$. All reactions were performed at $37{ }^{\circ} \mathrm{C}$ in the presence of $2 \mathrm{mM}$ ATP and $10 \mathrm{mM} \mathrm{MgCl}_{2} .50 \mu \mathrm{M} \mathrm{E} \mathrm{S}_{1} \mathrm{~S}, 100 \mu \mathrm{M}$ sodium cholate, saturating amounts of Ko143, and a $3 \times$ Molar excess of 5D3-Fab, were used to assess their stimulating or inhibitory effects. The molar ratios of nanodisc-reconstituted ABCG2 and 5D3-Fab were varied to determine the functional stoichiometry. For ATPase assays in proteoliposomes, 
samples were FT and extruded as described above. ATPase rates were determined using linear regression in GraphPad Prism 7.

\section{Cryo-EM data acquisition}

Cryo-EM grids of nanodisc-reconstituted ABCG2-5D3(Fab) were prepared using a Vitrobot Mark IV (FEI) with the environmental chamber set at $95 \%$ humidity and $10{ }^{\circ} \mathrm{C}$. Aliquots $(3 \mu \mathrm{L})$ of the complex, at a concentration of $\sim 0.2 \mathrm{mg} \mathrm{mL}^{-1}$, were placed on glow-discharged Quantifoil carbon grids (300 mesh, copper). Grids were blotted for $1.5 \mathrm{~s}$ and flash-frozen in a mixture of liquid ethane and propane cooled by liquid nitrogen. Grids were imaged with a Titan Krios (FEI) electron microscope that operated at $300 \mathrm{keV}$ equipped with a Quantum-LS energy filter and a K2 Summit electron counting direct detection camera (Gatan Co.). Images were recorded using SerialEM ${ }^{53}$ in super-resolution mode using a $20 \mathrm{eV}$ slit width of the GIF energy filter at a nominal magnification of $130,000 \times$, resulting in a calibrated super-resolution pixel size of $0.5196 \AA$ (physical pixel size $1.039 \AA$ ). Defocus was set to vary from -1.5 to $-2 \mu \mathrm{m}$. The super-resolution micrographs were down-sampled $2 \times$ by Fourier-cropping and drift-corrected and dose-weighted using MotionCor $2^{54}$ through the Focus suite (Biyani et al, manuscript in preparation). Each image was dose-fractionated to 40 frames ( $16 \mathrm{~s}$ exposure in total, $0.4 \mathrm{~s}$ per frame). The dose rate was set to $\sim 4.5 \mathrm{e}-/ \mathrm{pix} / \mathrm{s}(\sim 1.7$ $\mathrm{e}^{-} / \AA^{2} /$ frame) leading to a total dose of $\sim 67 \mathrm{e}^{-} / \AA^{2}$ (Extended Data Table 1). Focus was used to remove bad micrographs manually from the dataset.

\section{Image processing}

The drift-corrected, dose-weighted micrographs were imported in RELION-2.02-beta ${ }^{55-57}$ that was used for all data processing except where specified. CTF parameters were estimated with GCTF ${ }^{58}$. Micrographs had a calculated defocus of -1.1 to $-2.5 \mu \mathrm{m}$. Particles were picked in template-free manner using Gautomatch ${ }^{59} .92,326$ particles were selected from 1,702 electron micrographs. After three rounds of 2D classification and selection of particles belonging to "good" classes, the dataset was reduced to 32,806 particles. These particles were used to generate a model with 3D auto-refine. The resolution of this initial map was $5.9 \AA$. This map was then filtered to $20 \AA$ and projected in evenly spaced $\left(15^{\circ}\right)$ directions. Using these projections as templates, a total of 232,608 particles 
could be picked from the dataset. After three steps of 2D classification and selection we obtained a final dataset of 97,612 particles. Refinement of these particles against the same starting model as before using the 3D auto-refine procedure resulted in a 4.2 Å map. After post-processing (including soft masking and B-factor sharpening with the automatically determined B-factor of $-142.3 \AA^{2}$ ) in RELION-1.4 $4^{55,56}$, a map with a resolution of $3.8 \AA$ (by Fourier shell correlation using the $0.143 \AA$ cut-off criterion ${ }^{60-62}$ ) was obtained. To assist modelling, a local resolution-filtered map was calculated with RELION-2.02-beta (using a B-factor of -142.3 $\AA^{2}$ ).

\section{Model building and refinement}

For model building, we used mainly the post-processed map, but also the local resolution-filtered map and the original refinement map. The Coot program ${ }^{63}$ was used for all modelling steps. 5D3Fab was placed as a rigid body followed by separate fitting of the variable domain (VD) and constant domain (CD). A half-transporter atomic model for ABCG2 was generated using the HHpred server with MODELLER ${ }^{64}$, using the ABCG5 X-ray structure ${ }^{18}$ (chain A of PDB entry: 5DO7). The TMD and NBD domains were fitted separately in the electrostatic potential map. The half-transporter atomic coordinates were then duplicated and rigid-body docked into the region of the map corresponding to the other half of ABCG2 to complete the full transporter model.

The post-processed map was of excellent quality and allowed de novo model building (Extended Data Fig. 4) of the ABCG2-TMD and the VD of the 5D3-Fab. For the CD of 5D3-Fab we were especially careful when adapting the model to the map and took advantage of the local-resolution filtered map. In the region of the ABCG2-NBDs the post-processed map was of lower quality than in the regions of ABCG2-TMD and 5D3-Fab. A similar reduced resolution of NBDs was recently observed in the cryo-EM structures of the zebra fish CFTR protein and human TAP ${ }^{65,66}$. Given the lower resolution of the density covering the NBDs, we generated an NBD model based on the crystal structure of $\mathrm{ABCG} 5 / \mathrm{G} 8$, which shares $\sim 40 \%$ sequence identity in the NBD region. Upon docking this model into the density, we could adjust the position of the secondary structure elements by fitting them into the density. We used the local-resolution filtered map and the original non post-processed map for very conservative adjustment of the model, guided by the structures of ABCG5 and ABCG8 (chain A and B, respectively, of PDB entry 5DO7), the HHpred models using these structures as a template, as well as HHpred models based on the structures of Thermotoga 
maritima TM_0544 (PDB entry 1VPL), Alicyclobacillus acidocaldarius CysA $^{67}$ (chain A of PDB entry 1Z47), Thermotoga maritima TM_1403 (chain A of PDB entry 4YER), Geobacillus stearothermophilus ArtP in complex with $\mathrm{ADP} / \mathrm{Mg}^{2+}$ (chain A of PDB entry 2OLJ) and Thermoanaerobacter tengcongensis $\mathrm{CbiO}^{68}$ (chain $\mathrm{B}$ of $\mathrm{PDB}$ entry $4 \mathrm{MKI}$ ). We then placed two cholesterol molecules in cavity 1 of the ABCG2-TMD in the orientation that resulted in the best visual fit to the map. Finally, the complete ABCG2-5D3(Fab) atomic model was refined against the local resolution-filtered map (employing a resolution limit of $3.8 \AA$ ) with phenix.real_space_refine ${ }^{69}$. For the final round of model refinement we performed global realspace refinement with standard geometry restraints as well as rotamer, Ramachandran plot, C-beta, NCS and secondary structure restraints, coupled to reciprocal-space refinement of the B-factors. Refinement was performed including all side chains and with both cholesterol molecules. Refinement statistics of that model are given in Extended Data Table 1.

For validation of the refinement, random shifts (up to $0.3 \AA$ ) were introduced into the coordinates of the final refined model, followed by refinement with phenix.real_space_refine (using the same parameters as described before) against the first unfiltered half-map (half-map 1). The small "gap" between the FSC curve of the model with random displacements refined against half-map 1 versus half-map 1 and the FSC curve of the same model versus half-map 2 (against which it was not refined) indicates no over-refinement took place (Extended Data Figure $2 \mathrm{~g})^{70,71}$.

\section{Orientation determination of YFP-3C-ABCG2 in proteoliposomes}

ABCG2 was N-terminally tagged with yellow fluorescent protein (YFP) and 3C protease (YFP3C-ABCG2) and was expressed, purified and reconstituted into liposomes as described above. The sample was divided into two (disrupted and non-disrupted). $1 \% \mathrm{v} / \mathrm{v}$ Triton-X-100 was added to the 'disrupted' sample while the equivalent volume of transport buffer was added to the 'nondisrupted' sample, and both were incubated for $30 \mathrm{~min}$ at room temperature. $3 \mathrm{C}$ was added to both samples to cleave the YFP and the samples were centrifuged at $100,000 \mathrm{~g}$. Supernatants were collected and analyzed by reducing SDS-PAGE in-gel fluorescence using ImageJ.

\section{Pull-down assays of human and rat ABCG2 using 5D3-Fab}


Expression and solubilization of YFP-tagged human ABCG2 and of YFP-tagged rat ABCG2 was performed as described above but without kifunensine treatment. Following ultracentrifugation, the supernatants were incubated with 5D3-mAb-coupled sepharose resin. The resin was washed extensively with purification buffer and analyzed by reducing SDS-PAGE in-gel fluorescence.

\section{Expression, purification, crystallization and structure determination of the 5D3-Fab}

5D3 hybridoma cells, producing the 5D3 monoclonal antibody (mAb), were obtained from Brian Sorrentino ${ }^{16}$ and cultured in WHEATON CELLine Bioreactors according to the manufacturer's protocol. 5D3-Fab was purified from the supernatant as described in the Fab Preparation Kit protocol (Thermo Fisher Scientific). The purity and yield of 5D3-Fab were evaluated by reducing and non-reducing SDS-PAGE and the purified protein was crystallized in $100 \mathrm{mM}$ Tris $\mathrm{pH} 8.5$ and $16 \%$ PEG 6000 at $20^{\circ} \mathrm{C}$. A single 5D3-Fab crystal was mounted at $100 \mathrm{~K}$ on the goniometer of beamline PXI at the Swiss Light Source (SLS) and a complete dataset was collected to a maximum resolution of $1.5 \AA$ (Extended Data Table 2). The 5D3-Fab X-ray structure was determined by molecular replacement in Phaser $^{72}$ using the Fab of the PDB entry 4CAD as the search model ${ }^{73}$. Iterative model building was performed in $\mathrm{Coot}^{74}$ and the X-ray structure was refined in PHENIX ${ }^{69}$.

\section{Figure preparation}

Figures were prepared using the programs PyMOL (The PyMOL molecular graphics system DeLano Scientific) and Chimera ${ }^{75}$.

\section{Data availability}

Atomic coordinates of the ABCG2-5D3(Fab) model after truncating the side chains of the NBD and of the 5D3-Fab CD to alanine, have been deposited in the PDB under accession 5NJ3. The de novo built structure of the ABCG2 TMD in complex with 5D3-Fab VD was separately deposited in the PDB under accession 5NJG. Atomic coordinates of the X-ray structure of 5D3-Fab have been deposited in the PDB under accession 5NIV and the three-dimensional cryo-EM density postprocessed, masked map was deposited to the Electron Microscopy Data Bank under accession code EMD-3654.

49 Geisse, S., Jordan, M. \& Wurm, F. M. Large-scale transient expression of therapeutic proteins in mammalian cells. Methods in molecular biology 308, 87-98, (2005). 
50 Geertsma, E. R., Nik Mahmood, N. A., Schuurman-Wolters, G. K. \& Poolman, B. Membrane reconstitution of $\mathrm{ABC}$ transporters and assays of translocator function. Nature protocols 3, 256-266, (2008).

51 Schaffner, W. \& Weissmann, C. A rapid, sensitive, and specific method for the determination of protein in dilute solution. Analytical biochemistry 56, 502-514, (1973).

52 Chifflet, S., Torriglia, A., Chiesa, R. \& Tolosa, S. A method for the determination of inorganic phosphate in the presence of labile organic phosphate and high concentrations of protein: application to lens ATPases. Analytical biochemistry 168, 1-4, (1988).

53 Mastronarde, D. N. Automated electron microscope tomography using robust prediction of specimen movements. Journal of structural biology 152, 36-51, (2005).

$54 \mathrm{Li}, \mathrm{X}$. et al. Electron counting and beam-induced motion correction enable near-atomicresolution single-particle cryo-EM. Nature methods 10, 584-590, (2013).

55 Scheres, S. H. A Bayesian view on cryo-EM structure determination. Journal of molecular biology 415, 406-418, (2012).

56 Scheres, S. H. RELION: implementation of a Bayesian approach to cryo-EM structure determination. Journal of structural biology 180, 519-530, (2012).

57 Kimanius, D., Forsberg, B. O., Scheres, S. H. \& Lindahl, E. Accelerated cryo-EM structure determination with parallelisation using GPUs in RELION-2. eLife 5, (2016).

Zhang, K. Gctf: Real-time CTF determination and correction. Journal of structural biology 193, 1-12, (2016).

59 Urnavicius, L. et al. The structure of the dynactin complex and its interaction with dynein. Science 347, 1441-1446, (2015).

60 Rosenthal, P. B. \& Henderson, R. Optimal determination of particle orientation, absolute hand, and contrast loss in single-particle electron cryomicroscopy. Journal of molecular biology 333, 721-745, (2003).

61 Scheres, S. H. \& Chen, S. Prevention of overfitting in cryo-EM structure determination. Nature methods 9, 853-854, (2012).

62 Chen, S. et al. High-resolution noise substitution to measure overfitting and validate resolution in 3D structure determination by single particle electron cryomicroscopy. Ultramicroscopy 135, 24-35, (2013).

63 Emsley, P., Lohkamp, B., Scott, W. G. \& Cowtan, K. Features and development of Coot. Acta crystallographica. Section D, Biological crystallography 66, 486-501, (2010).

64 Webb, B. \& Sali, A. Protein structure modeling with MODELLER. Methods in molecular biology 1137, 1-15, (2014).

65 Zhang, Z. \& Chen, J. Atomic Structure of the Cystic Fibrosis Transmembrane Conductance Regulator. Cell 167, 1586-1597 e1589, (2016).

66 Oldham, M. L., Grigorieff, N. \& Chen, J. Structure of the transporter associated with antigen processing trapped by herpes simplex virus. eLife 5, (2016).

67 Scheffel, F. et al. Structure of the ATPase subunit CysA of the putative sulfate ATPbinding cassette $(\mathrm{ABC})$ transporter from Alicyclobacillus acidocaldarius. FEBS letters 579, 2953-2958, (2005).

68 Chai, C. et al. Structural basis for a homodimeric ATPase subunit of an ECF transporter. Protein \& cell 4, 793-801, (2013).

69 Adams, P. D. et al. PHENIX: a comprehensive Python-based system for macromolecular structure solution. Acta crystallographica. Section D, Biological crystallography 66, 213221, (2010). 
70 Brown, A. et al. Tools for macromolecular model building and refinement into electron cryo-microscopy reconstructions. Acta crystallographica. Section D, Biological crystallography 71, 136-153, (2015).

71 Fischer, N. et al. The pathway to GTPase activation of elongation factor SelB on the ribosome. Nature 540, 80-85, (2016).

72 McCoy, A. J. et al. Phaser crystallographic software. Journal of applied crystallography 40, 658-674, (2007).

73 Manolaridis, I. et al. Mechanism of farnesylated CAAX protein processing by the intramembrane protease Rce1. Nature 504, 301-305, (2013).

74 Emsley, P. \& Cowtan, K. Coot: model-building tools for molecular graphics. Acta crystallographica. Section D, Biological crystallography 60, 2126-2132, (2004).

75 Pettersen, E. F. et al. UCSF Chimera--a visualization system for exploratory research and analysis. Journal of computational chemistry 25, 1605-1612, (2004). 
Figure 1 | Transport and ATPase activities of liposome-reconstituted ABCG2. a, Schematic of ATP-driven transport of estrone-3-sulfate $\left(\mathrm{E}_{1} \mathrm{~S}\right)$. 5D3-Fab (depicted as yellow surface) inhibits $\mathrm{E}_{1} \mathrm{~S}$ transport only when bound to the extracellular side of $\mathrm{ABCG} 2$ in the lumen of the proetoliposomes. b, Normalized initial $\mathrm{E}_{1} \mathrm{~S}$ transport rates. $\mathrm{G} 2$ denotes $\mathrm{ABCG} 2$, FT denotes freezethawing allowing 5D3-Fab access to the liposome lumen. c, $\mathrm{E}_{1} \mathrm{~S}$-stimluated ATPase activity of ABCG2. In $\mathbf{b}$ and $\mathbf{c}$, the mean is plotted and error bars denote s.d. $(n \geq 3)$.

Figure 2 | ABCG2 structure. a, Ribbon diagram of ABCG2-5D3(Fab) complex. Protein subunits are labeled. G2' refers to the second ABCG2 monomer. b, ABCG2 structure without 5D3-Fab, with one monomer colored salmon. Motifs and residues associated with single nucleotide polymorphisms are colored blue and labeled. The other ABCG2 monomer is colored as a rainbow spectrum ranging from blue to red. c, Topology of the ABCG2 TMD colored as in $\mathbf{b}$. Cysteines forming inter- and intra-molecular disulfide bonds (C592, C603 and C608) are indicated, as is the N-glycosylated residue N596.

Figure 3 | ABCG2-5D3(Fab) interaction. a, Surface representation of ABCG2 and 5D3-Fab colored as in Fig. 2a. Black lines indicate membrane boundaries. b, Structure of the external loops EL3 and EL3' of the ABCG2 monomers and ribbon representation of the CDR regions of 5D3$\mathrm{Fab}$, as viewed from the extracellular side and colored as in $\mathbf{a}$. The inter- and intra-molecular disulfides formed by C592, C603 and C608 are shown as sticks, as is N596 and attached GlcNAc moieties. c, Representative SDS-PAGE of purified ABCG2-5D3(Fab) complex. The same sample was applied after (lane 1) or before (lane 2) disulfide reduction using DTT, demonstrating disulfide-dependent dimerization of ABCG2 (black arrows) and 5D3-Fab (red arrows). M denotes marker proteins, with selected masses indicated on the left. d, ATPase activity of nanodiscreconstituted $\mathrm{ABCG} 2$ at varying 5D3-Fab:ABCG2 ratios. The mean is plotted and error bars denote s.d. $(n=3)$. e, Partial sequence alignment of human and rat ABCG2. The seqeuence corresponding to EL3 is boxed, red asterisks depict cysteines involved in inter- and intra-molecular disulfides, green bars denote the ABCG2 EL3-5D3(Fab) interaction surfaces, and branches represent Nglycans.

Figure 4 | Translocation pathway. a, Sagittal slice through a surface representation of the ABCG2 TMD with cavities 1 and 2 indicated and the leucine plug depicted by a dashed cirle. b, Electrostatic surface potential of cavity 1 viewed from the cytoplasm with NBDs removed. Two bound cholesterol molecules are shown as green sticks. Arrows indicate entry sites into cavity 1 from the inner leaflet of the membrane. c, Ribbon diagram of the TMDs of ABCG2, with bound cholesterol molecules shown as green sticks and labeled. Helices are numbered as in Fig. 2c. d, EM density (white surface) around cholesterol molecule bound between TM2 and TM5a'. Residues within 4 
$\AA$ of bound cholesterol are shown as sticks and labeled. Panels a, $\mathbf{c}$ and $\mathbf{d}$ are colored as in Fig. 2a. e, f, Sequence alignments of TM2 and TM5a regions of human ABCG- subfamily members. Residues within $4 \AA$ of the bound cholesterol are marked with red asterisks.

Figure 5 | Proposed transport mechanism. ABCG2 monomers are colored red and blue, with cysteines forming disulfide bonds (yellow dashed lines) labeled "s". ATP-driven transport of substrate (orange hexagon labeled "S") occurs between states 1 and 2 (states indicated by circled numbers). Substrate enters cavity 1 (labeled "1") from the cytoplasm or from the inner leaflet of the membrane, but cannot access cavity 2 (labeled "2") due to the leucine plug (shown between ABCG2 monomers in states 1 and 3). Closing of the NBD interface converts state 1 to an outwardfacing state 2 that can release substrate to the outside. The release of inorganic phosphate (Pi) is accompanied by the conversion of ABCG2 from state 2 to state 1 . In state 3 (which corresponds to the structure presented here), two 5D3-Fabs (shaded green and yellow and labeled Fab) bind to the extracellular side of ABCG2. This leads to inhibition of transport and ATPase activity (indicated by red arrows to the left). While inhibition of transport occurs by clamping the ABCG2 monomers together, inhibiting ATP hydrolysis occurs allosterically (depicted by dashed red arrows). 
Figure 1

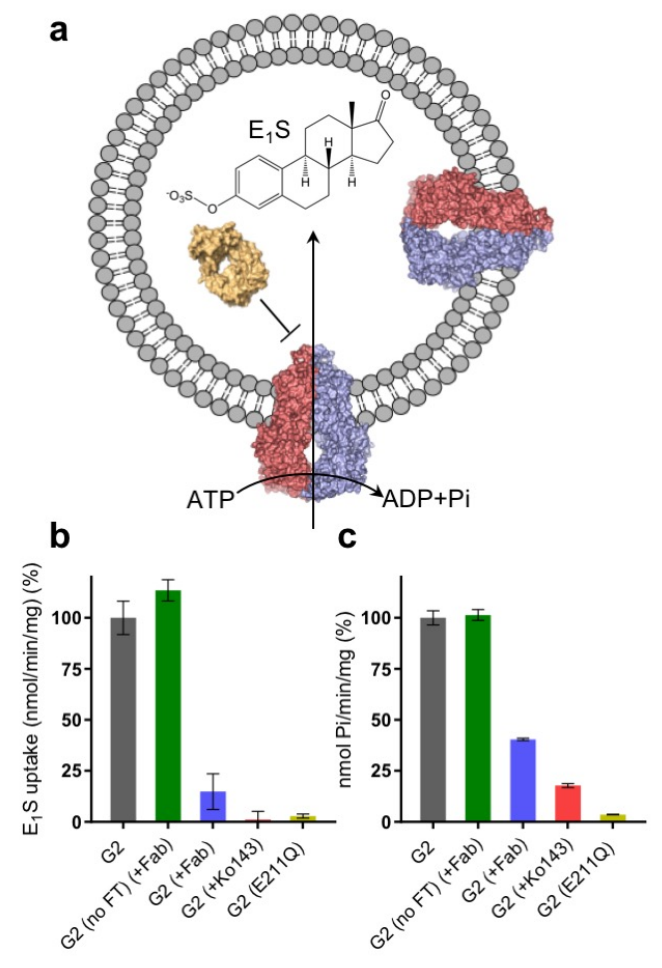


Figure 2

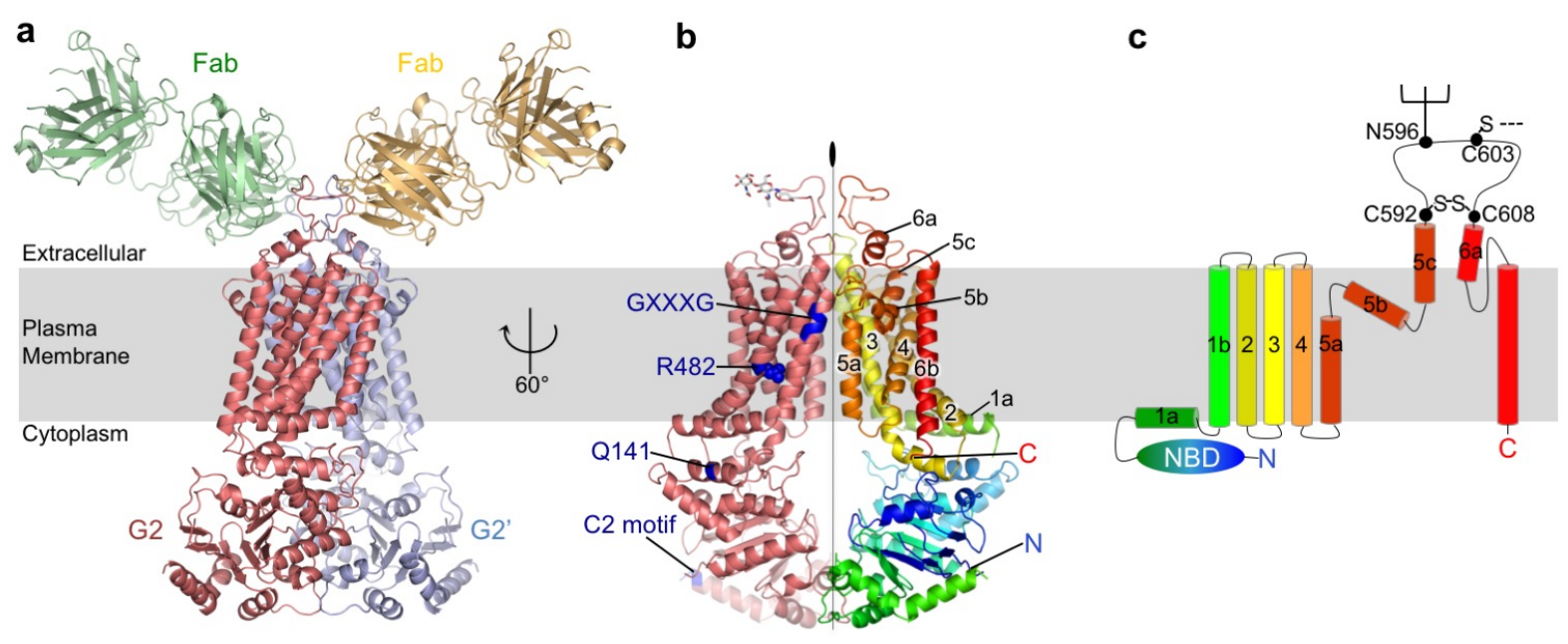


Figure 3
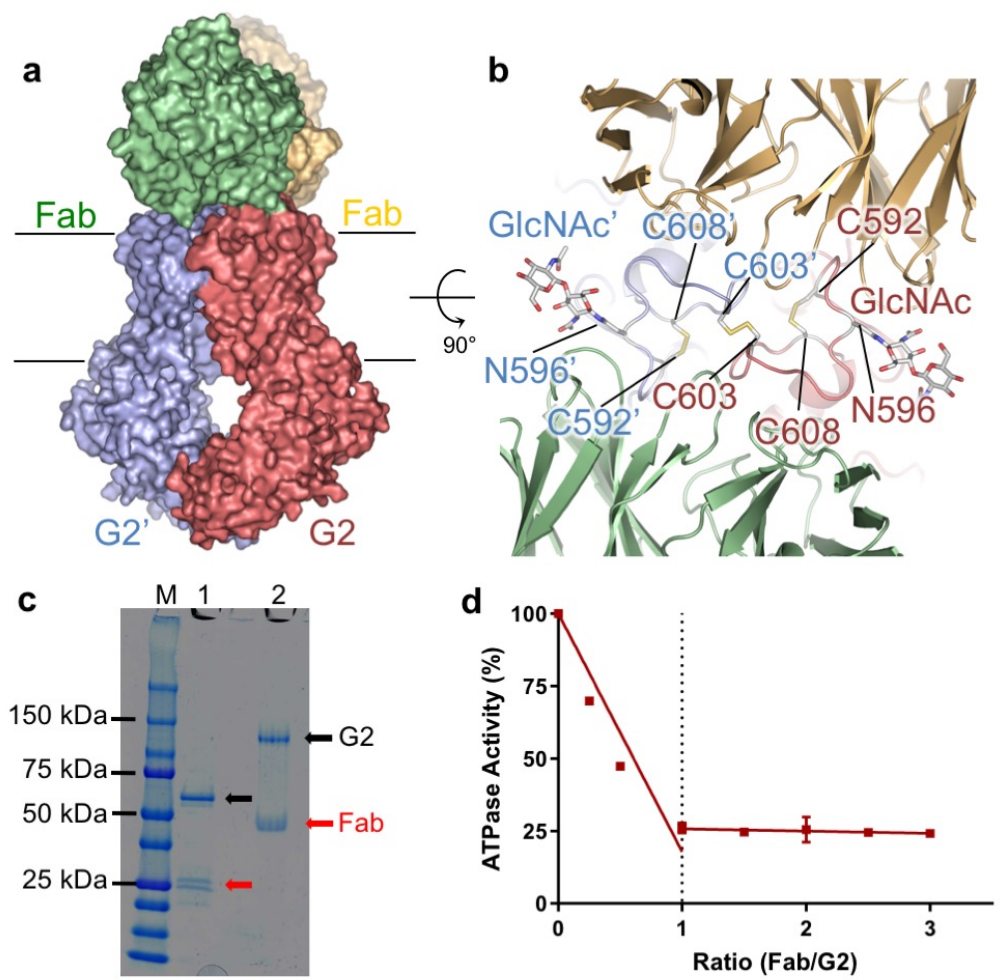

e

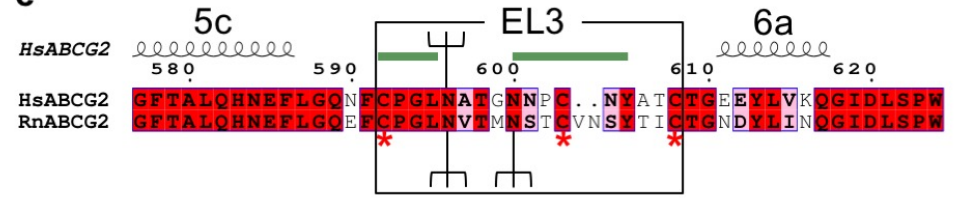




\section{Figure 4}
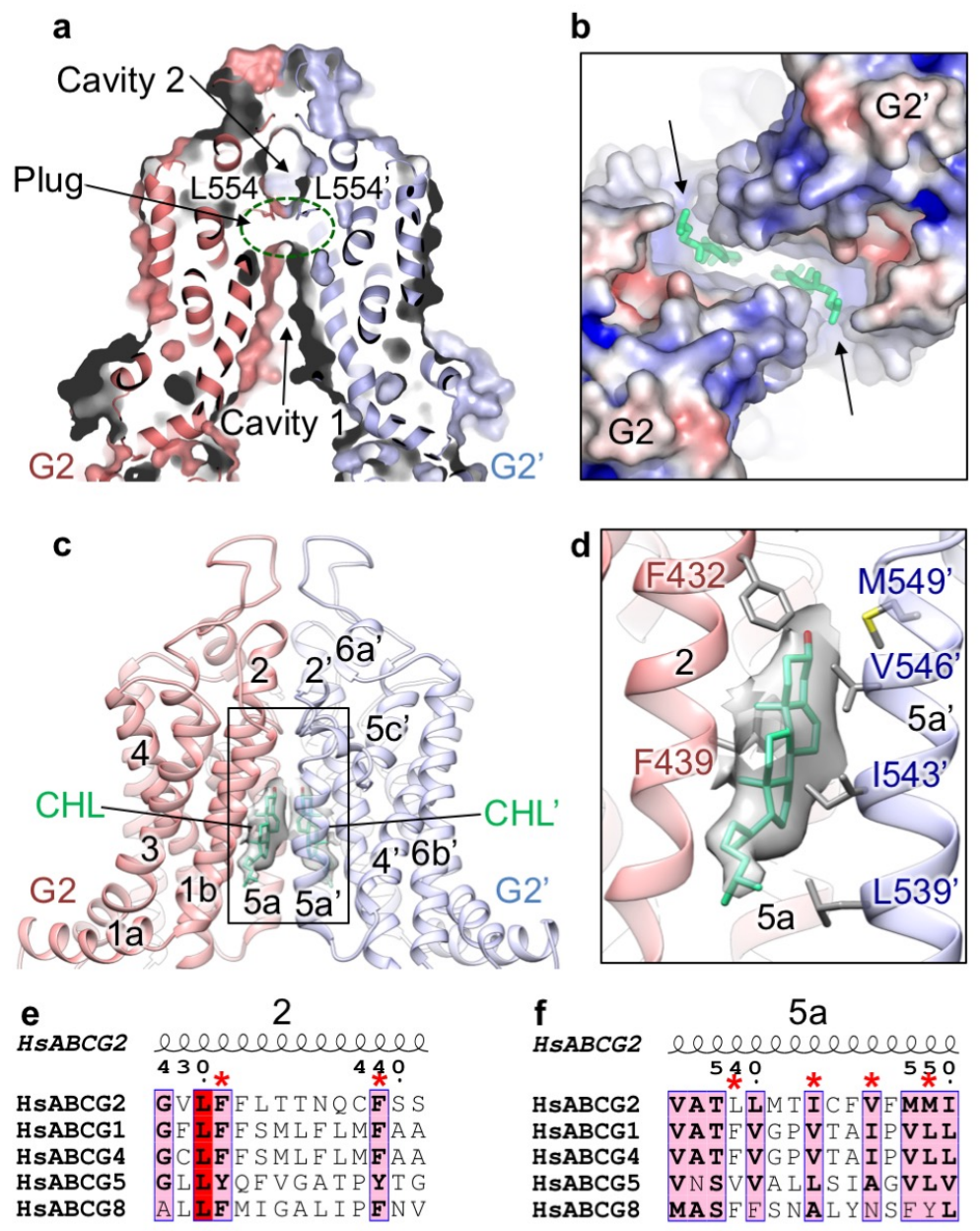

f

HsABCG2 lelelelelelele $540 *$ * 550

HSABCG2 VATLLMTICF HSABCG1 VATFVGPVTAIPVLI HSABCG4 VATFVGPVTAIPVII

HsABCG5 VNSVVALLS I A GVLV

HSABCG8 MASFESNALYNSEYL 
Figure 5

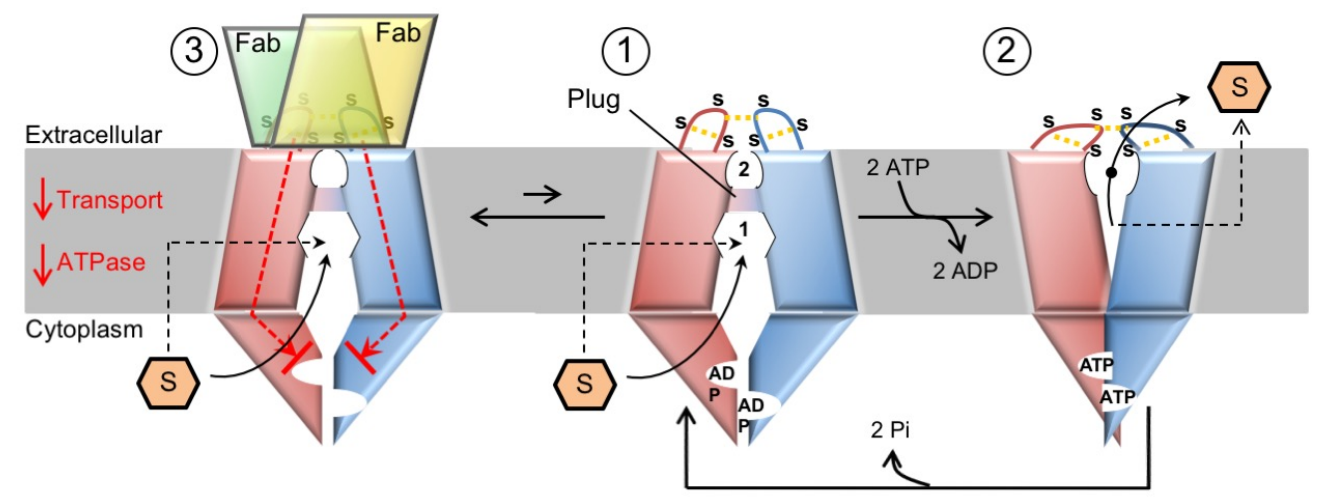



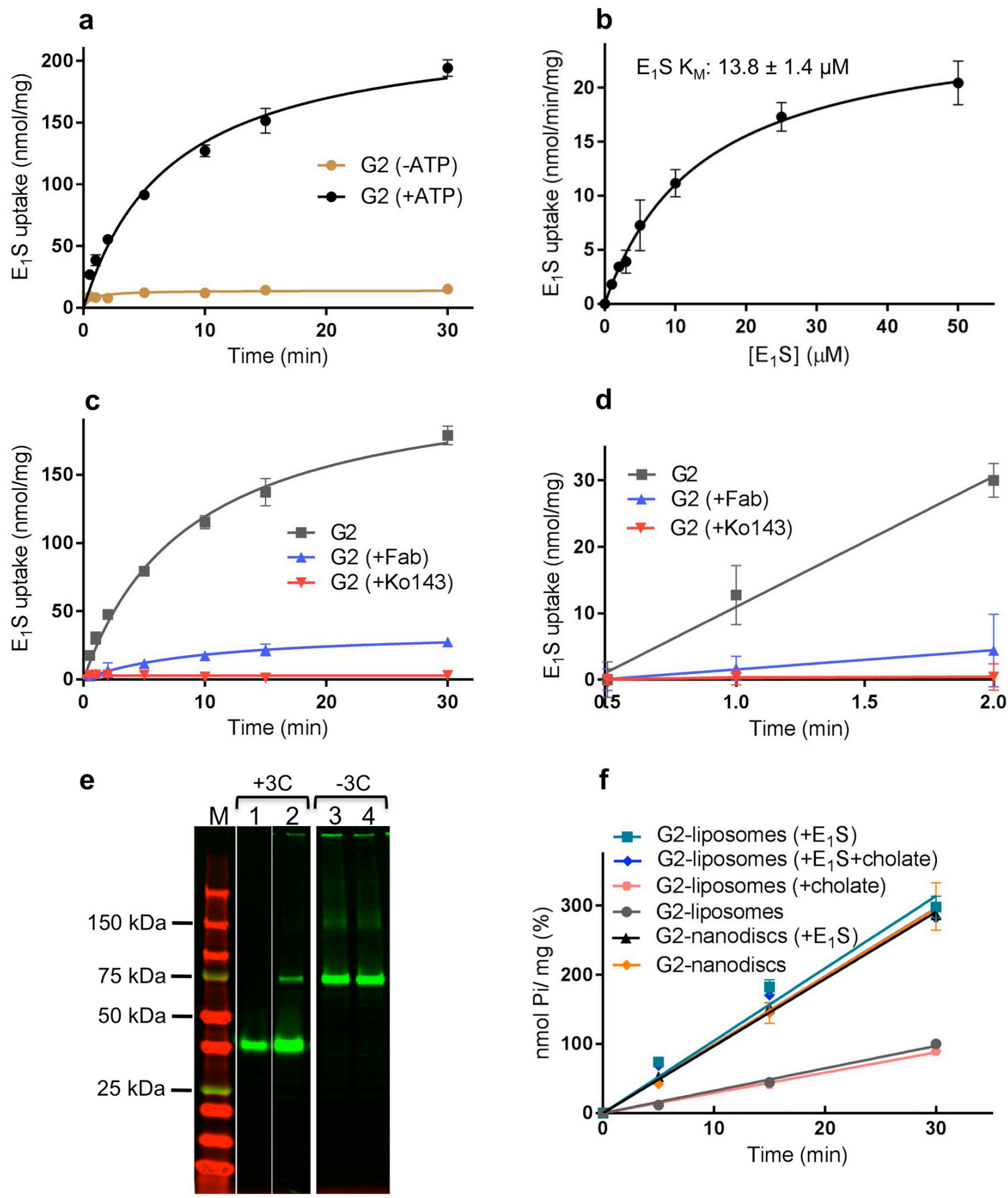

Extended Data Figure 1 | Functional studies of liposome-reconstituted ABCG2. a, Transport curves showing $E_{1} S$ uptake by $A B C G 2$ in the presence and absence of ATP. bo The rate of $E_{1} S$ transport was measured at different $\mathrm{E}_{1} \mathrm{~S}$ concentrations and the $\mathrm{K}_{\mathrm{M}}$ was determined. c, ATPdependent transport curves showing $\mathrm{E}_{1} \mathrm{~S}$ uptake in the presence and absence of Ko143 or 5D3Fab. d, The 30sec - 2 min linear portion of the curves from $\mathbf{c}$ used to determine the initial rates of 
$\mathrm{E}_{1} \mathrm{~S}$ transport. e, Representative SDS-PAGE to determine the orientation of liposome-reconstituted YFP-3C-ABCG2 using in-gel fluorescence, comparing the intensity of free YFP after the addition of $3 \mathrm{C}$ protease in disrupted and non-disrupted samples. $54 \pm 2 \%$ of ABCG 2 was oriented with the NBDs on the outside of the proteoliposomes and all assays were subsequently corrected for this value. The error denotes s.d. $(\mathrm{n}=3)$. Non-disrupted supernatant $(+3 \mathrm{C})$ (lane 1); disrupted supernatant (+3C) (lane 2); non-disrupted supernatant (-3C) (lane 3); disrupted supernatant (-3C) (lane 4). $\mathbf{f}$, The basal ATPase activity of nanodisc-reconstituted ABCG2 is equivalent to the $\mathrm{E}_{1} \mathrm{~S}-$ stimulated activity of liposome-reconstituted ABCG2 and cannot be stimulated further. ABCG2 is not responsive to cholate in the presence or absence of $E_{1} S$. The curves have been normalized to the basal ATPase activity of liposome-reconstituted ABCG2. In $\mathbf{a}-\mathbf{d}$ and $\mathbf{f}$ the mean is plotted and error bars denote s.d. $(\mathrm{n} \geq 3)$. 
a

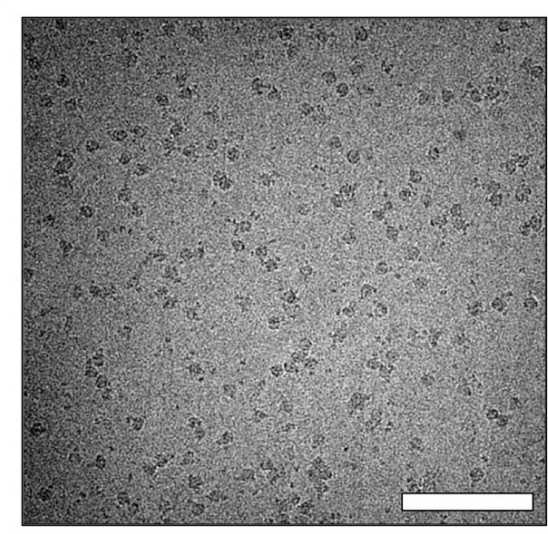

C

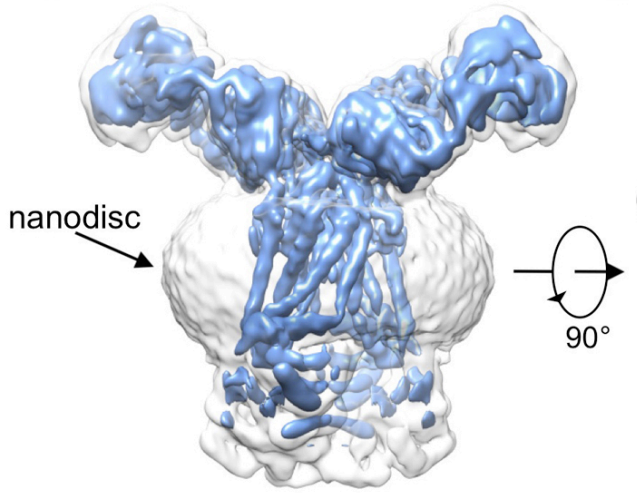

b

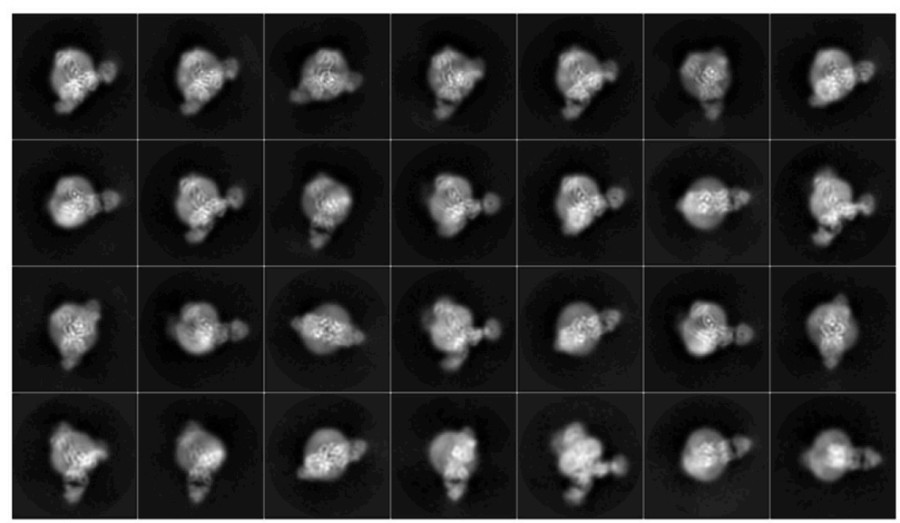

e
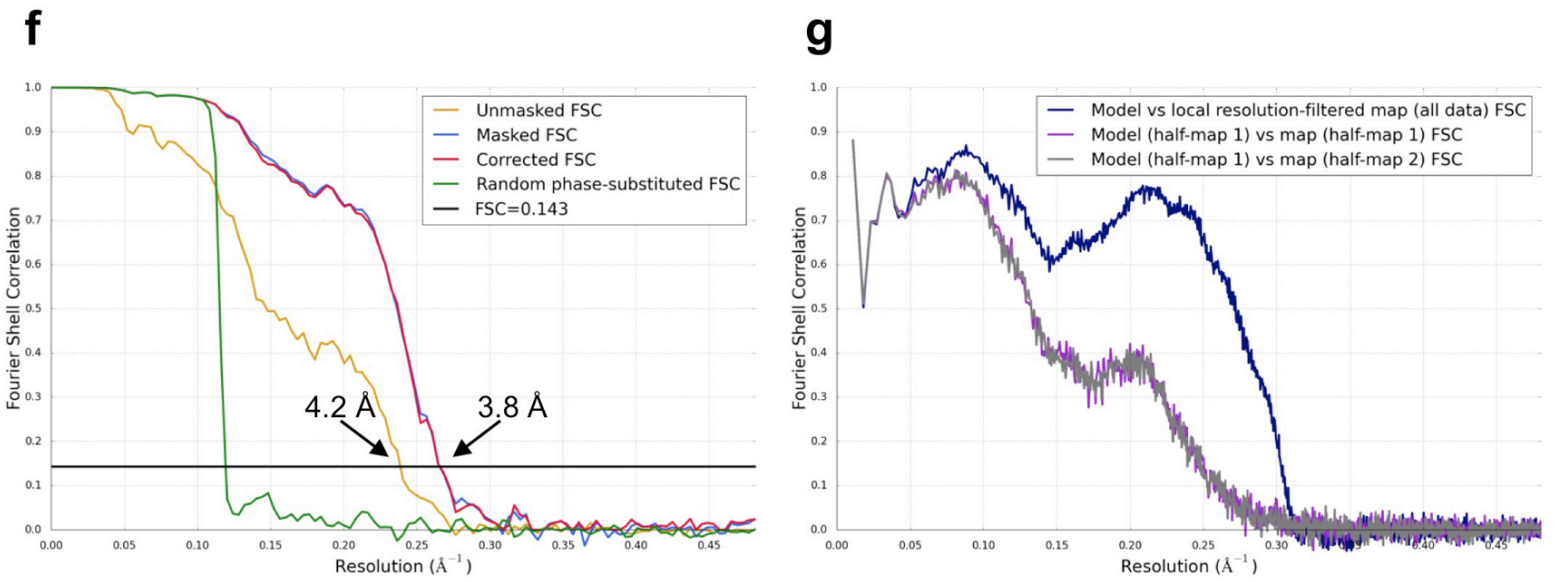

Extended Data Figure 2 | Cryo-EM map generation and atomic model refinement. a, An example micrograph (drift-corrected, dose-weighted and low-pass filtered to $20 \AA$ ) of the nanodiscreconstituted $\mathrm{ABCG} 2-5 \mathrm{D} 3(\mathrm{Fab})$ dataset. The white scale bar represents 1,000 $\AA$. b, The 28 most prevalent $2 \mathrm{D}$ class averages of the final round of $2 \mathrm{D}$ classification, sorted in decreasing order by the number of particles assigned to each class. c, Map obtained from the RELION 3D auto-refine procedure shown at a lower (white, transparent) and higher (blue, non-transparent) threshold. Density corresponding to the nanodisc is indicated with an arrow. d, Same as c but rotated. e, 
Angular distribution plot for the final reconstruction. The refinement map is shown at two different thresholds as in $\mathbf{c}$, in the same orientation. f, Fourier shell correlation from the Relion auto-refine procedure of the unmasked half-maps (yellow), the random-phase corrected half-maps (green), the half-maps after masking (blue) and the half-maps after masking and correction for the influence of the mask (red). A horizontal line (black) is drawn for the FSC $=0.143$ criterion. For both the unmasked and the corrected FSC curves, their intersection with the FSC $=0.143$ line is indicated by an arrow, and the resolution at this point is indicated. $\mathbf{g}$, Fourier shell correlation of the final model versus the map calculated with all data after local resolution-filtering, against which it was refined, (dark blue) and of the final model with introduced shifts (up to $0.3 \AA$ ) refined against the first unfiltered half-map (half-map 1) versus the same unfiltered half-map (violet) and versus the other unfiltered half-map (half-map 2) against which it was not refined (grey). 
a

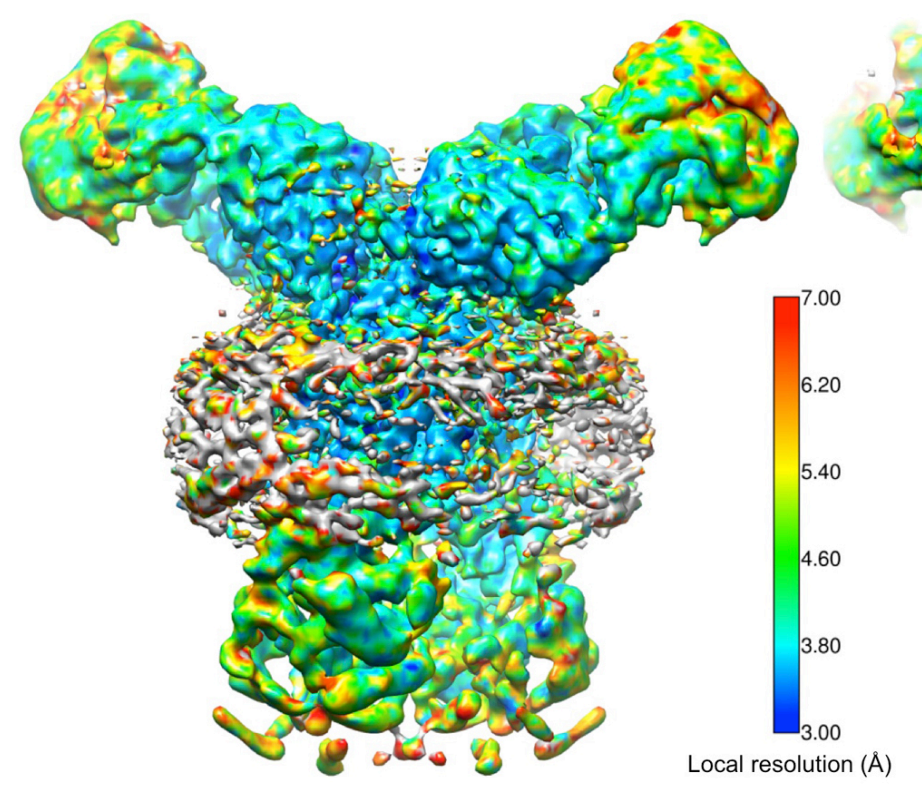

b

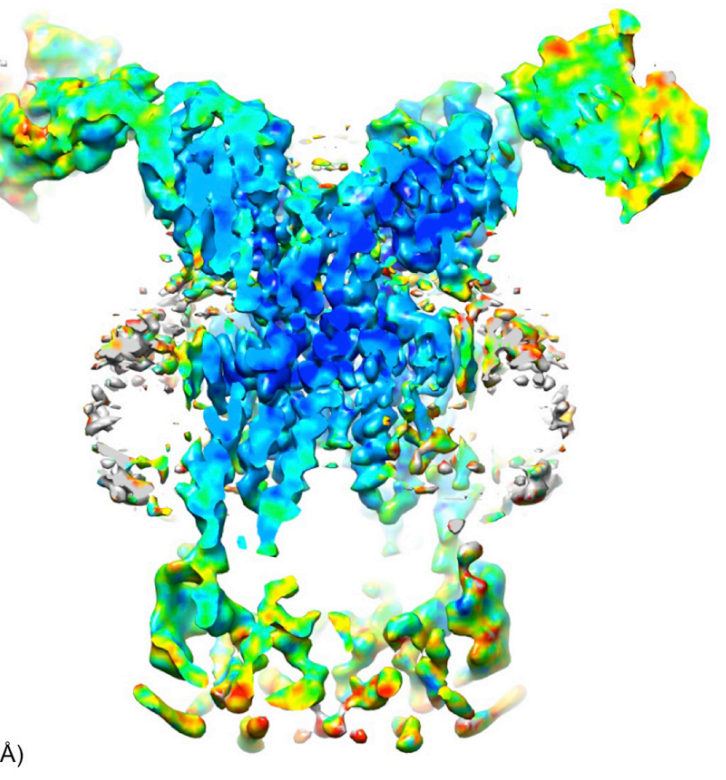

C

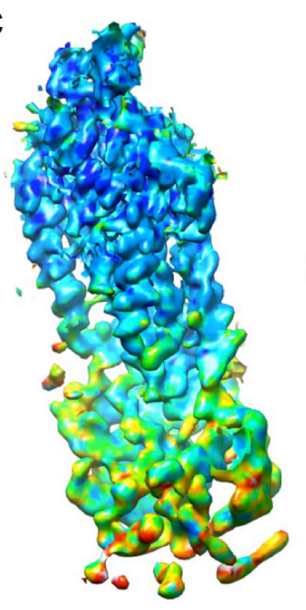

d

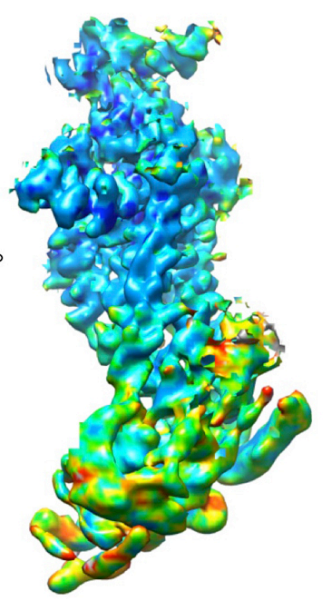

e

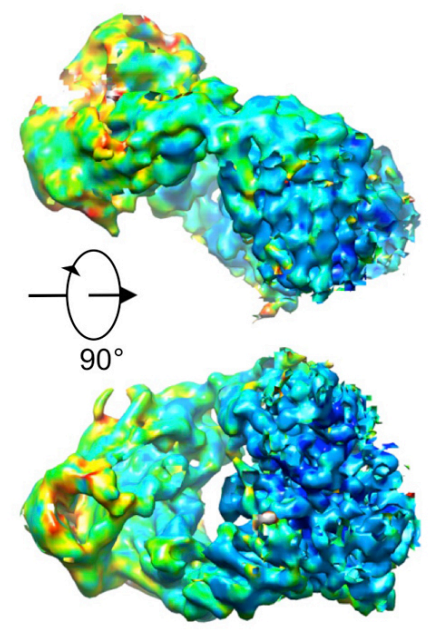

Extended Data Figure 3 | Local resolution. a, Full view of the RELION local resolution-filtered map colored by local resolution as calculated by ResMap. b, Same as a but only showing the posterior half (front clipping plane in the middle of the molecule). $\mathbf{c}$, Same as a but only showing a region up to $3 \AA$ around one chain of ABCG2 (including the putative cholesterol molecule and side chains of the NBD). d, Same as $\mathbf{c}$ but rotated. e, Same as a but only showing a region up to 3 $\AA$ around one 5D3-Fab. f, Same as e but rotated. 

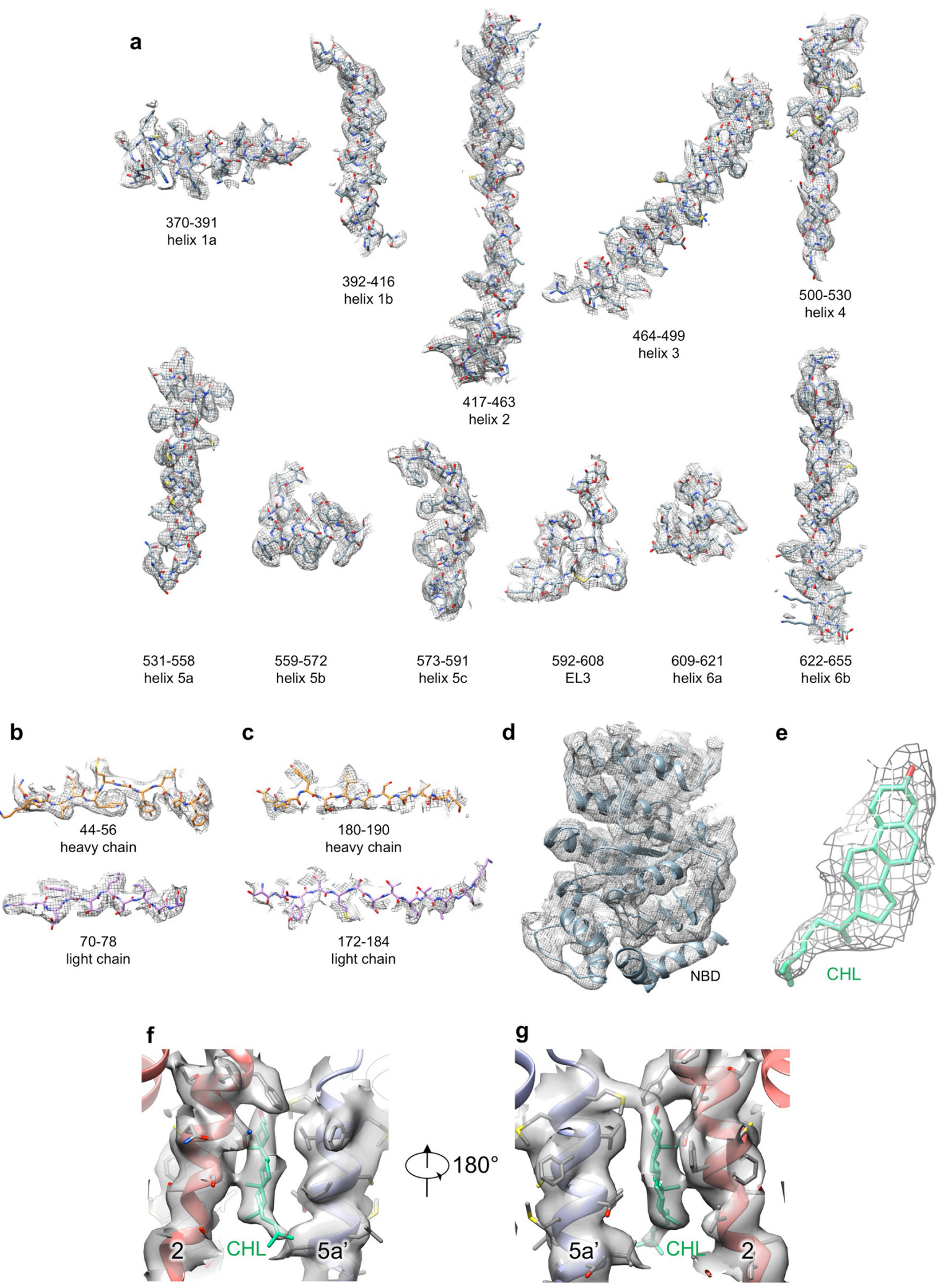

Extended Data Figure $4 \mid$ Fit of the model to the density. a, Fit of fragments of the final model of the ABCG2-TMD to the post-processed and masked map from RELION. A region of up to $2 \AA$ around the atoms is shown. The residue numbers are indicated, as are helices that are contained in 
these fragments and the EL3 b, Same as a but showing regions of the 5D3-Fab-VD. Residue numbers and whether the fragment belongs to the heavy or the light chain is indicated. c, Same as b but showing regions of the 5D3-Fab-CD. d, Fit of the ABCG2-NBD model to the local resolution-filtered map. A region of up to $3 \AA$ around one ABCG2-NBD (including side chains) is shown. e, Same as a but showing the cholesterol (CHL) molecule. f, Fit of fragments of ABCG2 TM2, TM5a and cholesterol (CHL) to the post-processed and masked map from RELION. A region of up to $2 \AA$ around the atoms is shown. $\mathbf{g}$, Same as $\mathbf{f}$ but rotated by $180^{\circ}$. 


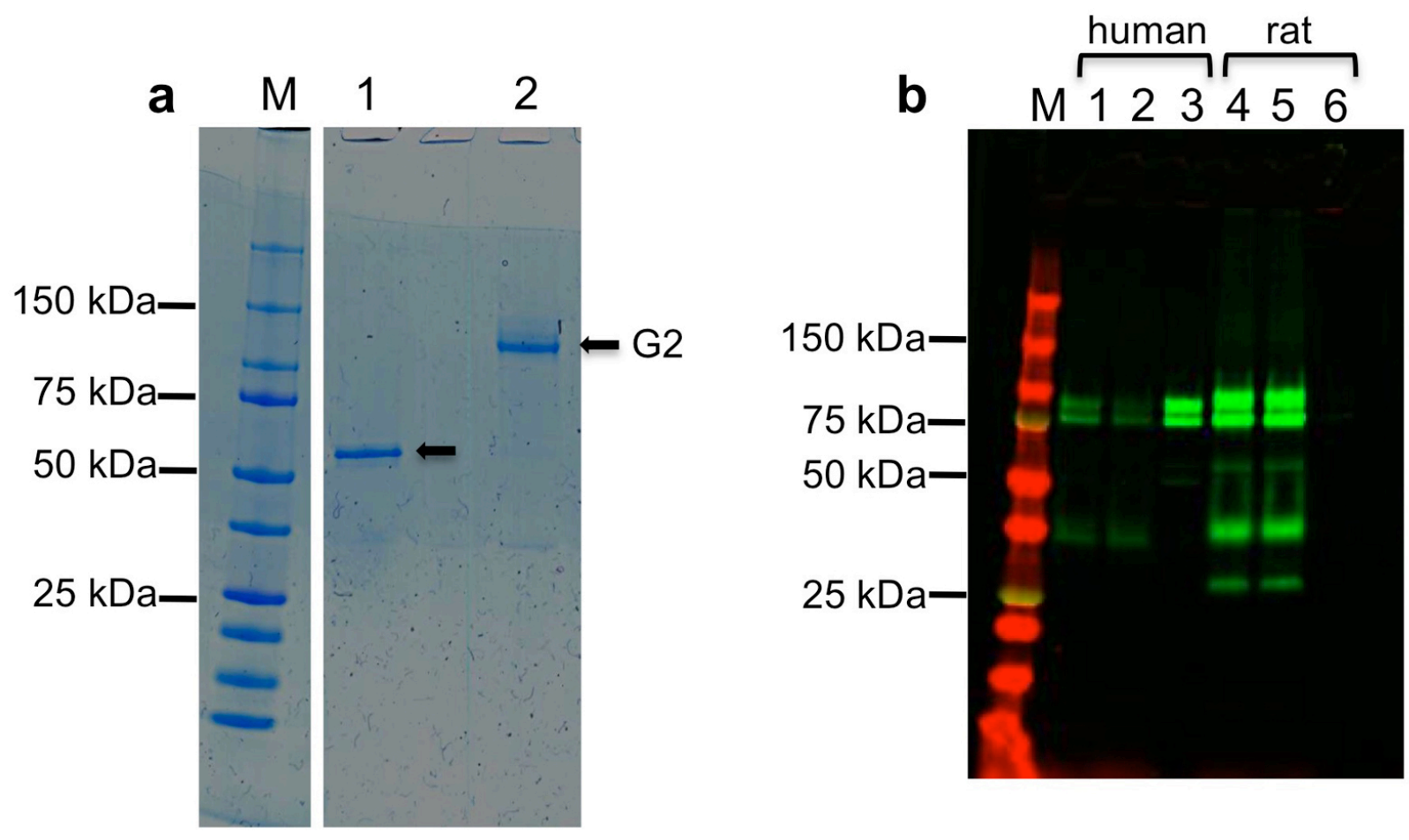

Extended Data Figure 5 | The X-ray structure and electrostatic surface potential of 5D3-Fab. a, Cartoon representation of the X-ray structure of 5D3-Fab. The light and heavy chain CDR1s are colored red, CDR2s are blue and CDR3s are purple. b, The molecular surface of 5D3-Fab, colorcoded by electrostatic potential ranging from blue (most positive) to red (most negative) to white (uncharged). 

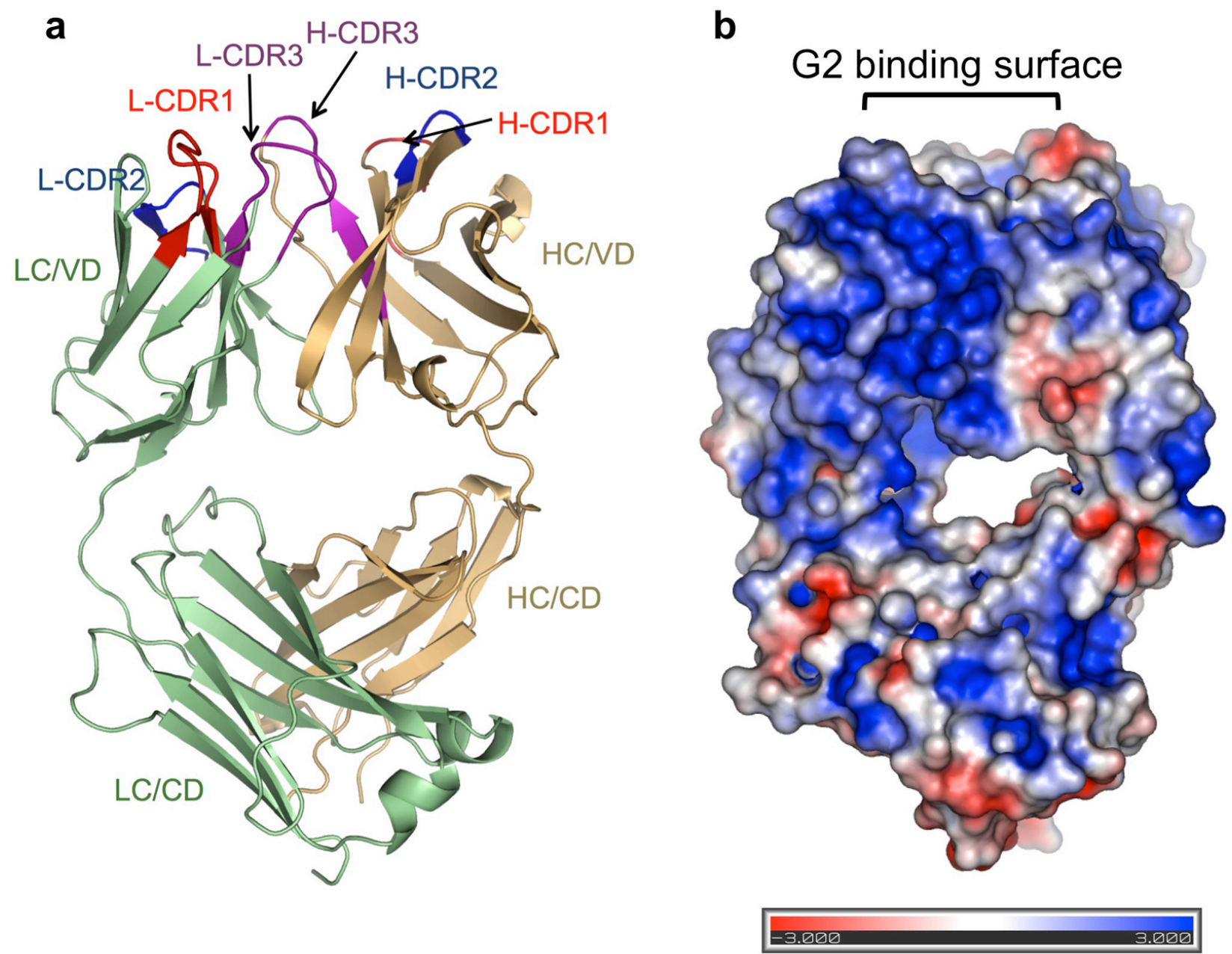

Extended Data Figure 6 | Purification of human ABCG2 and pull-down assays of human and rat ABCG2 using the 5D3 mAb. a, Representative SDS-PAGE of purified ABCG2. The same sample was applied after (lane 1) or before (lane 2) disulfide reduction using DTT, demonstrating disulfide-dependent dimerization of ABCG2 (black arrows). M indicates marker proteins, with selected masses indicated on the left. $\mathbf{b}$, Pull-down assay showing that the sepharose-immobilized 5D3-mAb does not bind to rat YFP-ABCG2. Human YFP-ABCG2 cell lysate (lane 1), flowthrough (lane 2) and elution (lane 3). Rat YFP-ABCG2 cell lysate (lane 4), flow-through (lane 5) and elution (lane 6). 
a

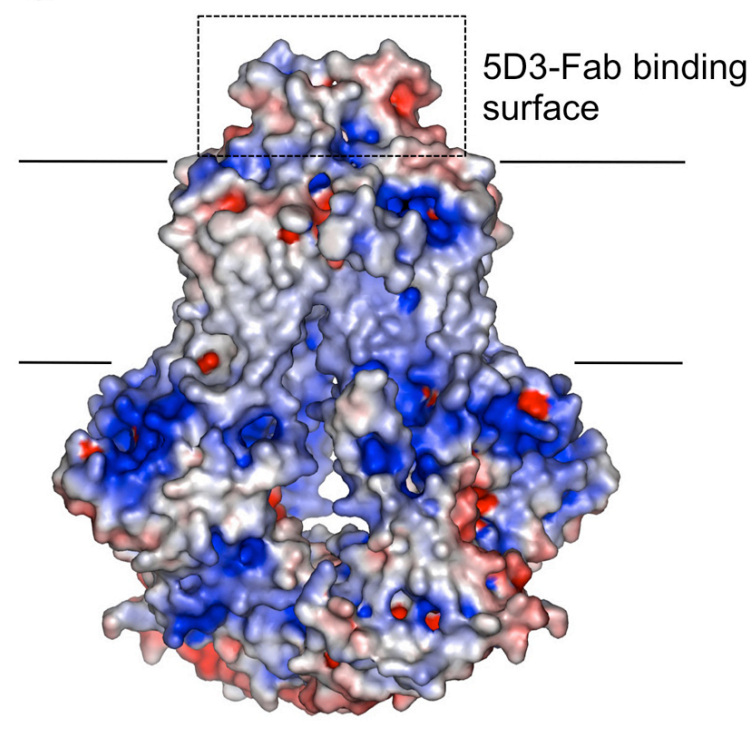

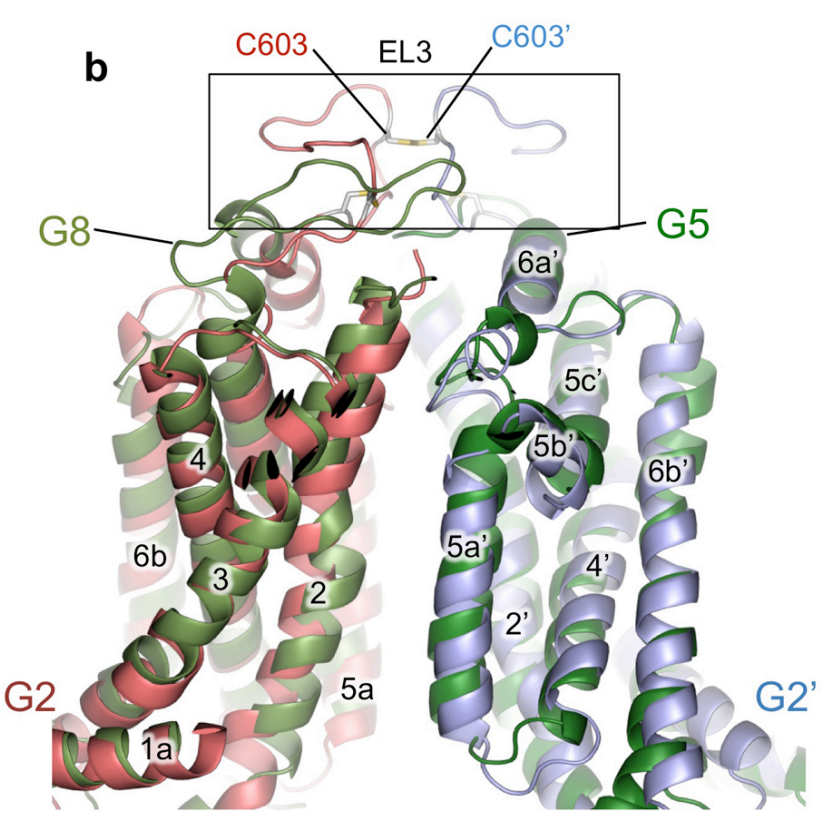

\section{EL3}

C

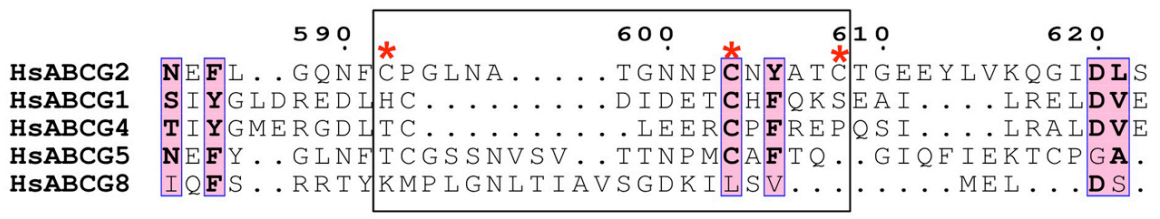

Extended Data Figure 7 | Electrostatic surface potential of ABCG2 and comparison of ABCG2 and ABCG5/G8. a, The molecular surface of ABCG2, color-coded by electrostatic potential ranging from blue (most positive) to red (most negative) to white (uncharged). The lipid membrane ( $40 \AA$ ) is indicated by the black lines based on the distribution of non-polar residues and TMs and the 5D3-Fab binding surface is boxed. b, Overlay of the ABCG2 and ABCG5/G8 TMDs with EL3 boxed. $\mathbf{a}$ and $\mathbf{b}$ are colored as in Fig. $2 \mathrm{a}$ and ABCG5/G8 is colored green. $\mathbf{c}$, EL3 sequence alignment of the G-family of ABC transporters with the cysteines involved in disulfide bond formation indicated by red asterisks and with EL3 boxed. 


\section{Extended Data Table 1. Summary of cryo-EM data}

\begin{tabular}{|c|c|}
\hline \multicolumn{2}{|l|}{ Cryo-electron microscopy data collection } \\
\hline Microscope & FEI Titan Krios \\
\hline Voltage $(\mathrm{kV})$ & 300 \\
\hline Camera & Gatan K2-Summit \\
\hline Energy Filter & Gatan Quantum-LS (GIF) \\
\hline Pixel size $(\AA)$ & 1.039 \\
\hline Defocus range $(\mu \mathrm{m})$ & $-1.1--2.5$ \\
\hline \multicolumn{2}{|l|}{ 3D reconstruction } \\
\hline Number of micrographs & 1,702 \\
\hline Final number of particles & 97,612 \\
\hline Resolution $(\AA)$ & 3.78 \\
\hline Sharpening B-factor $\left(\AA^{2}\right)$ & -142.3 \\
\hline \multicolumn{2}{|l|}{ Coordinate and B-factor refinement } \\
\hline Number of protein atoms (non-H) & 15,150 \\
\hline Number of ligand atoms (non-H) & 112 \\
\hline Mean B-factor protein atoms $\left(\AA^{2}\right)$ & 93.81 \\
\hline Mean B-factor of non-protein atoms $\left(\AA^{2}\right)$ & 69.47 \\
\hline RMSD bonds $(\AA)$ & 0.01 \\
\hline RMSD bond angles $\left({ }^{\circ}\right)$ & 1.15 \\
\hline Map CC (whole map) & 0.754 \\
\hline Map CC (around atoms) & 0.787 \\
\hline \multicolumn{2}{|l|}{ FSC (Model, Map) } \\
\hline Overall (entire box) & 0.763 \\
\hline Around atoms (masked) & 0.894 \\
\hline \multicolumn{2}{|l|}{ Ramachandran plot } \\
\hline Favored (\%) & 93.68 \\
\hline Allowed (\%) & 6.01 \\
\hline Disallowed (\%) & 0.31 \\
\hline \multicolumn{2}{|l|}{ Validation } \\
\hline Molprobity score & 1.93 \\
\hline All-atom clashscore & 9.64 \\
\hline Rotamer outliers (\%) & 0.36 \\
\hline
\end{tabular}

All statistics are for the final refinement model before the truncation of the ABCG2-NBD side chains to alanine and before the removal of the two cholesterol molecules. 


\section{Extended Data Table 2. Data collection and refinement statistics}

\begin{tabular}{|c|c|}
\hline \multicolumn{2}{|l|}{ 5D3-Fab X-ray structure } \\
\hline \multicolumn{2}{|l|}{ Data collection } \\
\hline Wavelength $(\AA)$ & 1.0 \\
\hline Resolution $(\AA)$ & $\begin{array}{l}41.50-1.50 \\
(1.55-1.50)\end{array}$ \\
\hline Space group & C 121 \\
\hline Unit cell & 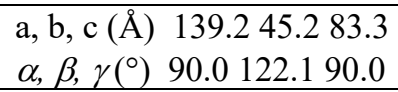 \\
\hline Total reflections & $468970(43093)$ \\
\hline Unique reflections & $66575(6208)$ \\
\hline Multiplicity & $7.0(6.9)$ \\
\hline Completeness (\%) & $94.1(88.2)$ \\
\hline Mean I/ $/(\mathrm{I})$ & $16.9(4.2)$ \\
\hline Wilson B-factor & 14.85 \\
\hline R-merge & $7.3(56.6)$ \\
\hline R-meas & $7.9(61.2)$ \\
\hline R-pim & $2.9(22.8)$ \\
\hline $\mathrm{CC} 1 / 2$ & $99.8(82.0)$ \\
\hline $\mathrm{CC}^{*}$ & $100(94.9)$ \\
\hline \multicolumn{2}{|l|}{ Refinement } \\
\hline Reflections used in refinement & $66574(6208)$ \\
\hline Reflections used for R-free & $3329(310)$ \\
\hline R-work & $18.0(30.2)$ \\
\hline R-free & $20.5(32.4)$ \\
\hline Number of non-hydrogen atoms & 3848 \\
\hline macromolecules & 3273 \\
\hline solvent & 575 \\
\hline Protein residues & 425 \\
\hline RMSD (bonds) ( $\AA$ ) & 0.006 \\
\hline RMSD (angles) $\left(^{\circ}\right)$ & 1.00 \\
\hline Ramachandran favored (\%) & 96.66 \\
\hline Ramachandran allowed (\%) & 2.86 \\
\hline Ramachandran outliers (\%) & 0.48 \\
\hline Rotamer outliers (\%) & 0.27 \\
\hline Clashscore & 9.16 \\
\hline Average B-factor & 19.83 \\
\hline macromolecules & 18.08 \\
\hline solvent & 29.74 \\
\hline
\end{tabular}

A single crystal was used for the 5D3-Fab structure

Statistics for the highest-resolution shell are shown in parentheses. 